\title{
Toda hierarchies and their applications
}

\author{
Kanehisa Takasaki* \\ Department of Mathematics, Kindai University \\ 3-4-1 Kowakae, Higashi-Osaka, Osaka 577-8502, Japan
}

\begin{abstract}
The 2D Toda hierarchy occupies a central position in the family of integrable hierarchies of the Toda type. The 1D Toda hierarchy and the Ablowitz-Ladik (aka relativistic Toda) hierarchy can be derived from the $2 \mathrm{D}$ Toda hierarchy as reductions. These integrable hierarchies have been applied to various problems of mathematics and mathematical physics since 1990s. A recent example is a series of studies on models of statistical mechanics called the melting crystal model. This research has revealed that the aforementioned two reductions of the 2D Toda hierarchy underlie two different melting crystal models. Technical clues are a fermionic realization of the quantum torus algebra, special algebraic relations therein called shift symmetries, and a matrix factorization problem. The two melting crystal models thus exhibit remarkable similarity with the Hermitian and unitary matrix models for which the two reductions of the 2D Toda hierarchy play the role of fundamental integrable structures.
\end{abstract}

2010 Mathematics Subject Classification: 17B65, 37K10, 82B20

Key words: Toda lattice, integrable hierarchy, free fermion, melting crystal, quantum torus, shift symmetry, factorization problem

*E-mail: takasaki@math.kindai.ac.jp 


\section{Introduction}

In 1967, Morikazu Toda introduced a one-dimensional lattice mechanical system with exponential interactions nowadays called the Toda lattice [1]. Though designed to have a periodic solution written in terms of elliptic functions [2], this nonlinear lattice was soon shown to have a solution with colliding solitons. This suggested remarkable similarity with the KdV equation, hence integrability.

Integrability of the Toda lattice was established by the middle of 1970s after the construction of exact $N$-soliton solutions [3, first integrals in involution [4, 5], Lax pairs for the inverse scattering method [6, 7] and finite-band integration of the periodic problem [8, 9]. These results were extended to a system of two-dimensional relativistic fields with exponential interactions among the components of the fields. By the end of 1970s, this 2D Toda field equation was proved to be integrable by the Lie group theory [10], the inverse scattering method [11, 12] and the bilinearization method [13]. In the beginning of $1980 \mathrm{~s}$, a fully 3D discretization was proposed in a bilinear form along with $N$-soliton solutions [13]. A description of more general solutions of this discrete system was soon presented in the language of a $2 \mathrm{D}$ complex free fermion system [14].

The Toda hierarchies [15] were developed as a Toda version of the KP hierarchy [16, 17] and its various relatives [18]. One of its prototypes is an unpublished result of van Moerbeke that is quoted in the work of Adler [19]. This result explains how to construct an integrable hierarchy of Lax equations for a difference operator $\mathfrak{L}$. In particular, the integrable hierarchy for the Jacobi operator

$$
\mathfrak{L}=e^{\partial_{s}}+b+c e^{-\partial_{s}},
$$

referred to as the $1 D$ Toda hierarchy, contains the equation of motion of the Toda lattice as the lowest member of the Lax equations with time variables $\boldsymbol{t}=\left(t_{1}, t_{2}, \ldots\right)$. In view of the construction of the KP hierarchy with a pseudo-differential Lax operator, it is natural to extend this construction to a "pseudo-difference operator" of the form

$$
L=e^{\partial_{s}}+u_{0}+u_{1} e^{-\partial_{s}}+\cdots .
$$

This extension, however, is not enough to accommodate the 2D Toda field equation. To this end, another Lax operator of the form

$$
\bar{L}^{-1}=\bar{u}_{0} e^{-\partial_{s}}+\bar{u}_{1}+\bar{u}_{2} e^{\partial_{s}}+\cdots
$$

has to be introduced along with another set $\overline{\boldsymbol{t}}=\left(\bar{t}_{1}, \bar{t}_{2}, \ldots\right)$ of time variables. The $2 D$ Toda hierarchy consists of Lax equations for these two Lax operators 
$L, \bar{L}$ with respect to the two sets $\boldsymbol{t}, \overline{\boldsymbol{t}}$ of time variables. The whole system of these Lax equations turns out to be equivalent to a system of ZakharovShabat equations for difference operators. The 2D Toda field equation is contained therein as the lowest member.

The 2D Toda hierarchy can be reformulated as a system of bilinear equations of the Hirota form for a single tau function $\tau(s, \boldsymbol{t}, \overline{\boldsymbol{t}}$ ) (in which the lattice coordinate $s$ is treated on an equal footing with the other independent variables). These bilinear equations can be cast into (and derived from) a generating functional form. One can thereby deduce [15] that $\tau(s, \boldsymbol{t}, \overline{\boldsymbol{t}})$, up to a sign factor, coincides with the tau function of the two-component KP hierarchy with charge $(s,-s)$ [20]. This leads to a fermionic formula of $\tau(s, \boldsymbol{t}, \overline{\boldsymbol{t}})$. Actually, $\tau(s, \boldsymbol{t}, \overline{\boldsymbol{t}})$ has another fermionic formula [21, 22, 23. that is directly related to a matrix factorization problem for solving the $2 \mathrm{D}$ Toda hierarchy in the Lax formalism [24]. This fermionic formula is a very powerful tool for studying various special solutions of the 2D Toda hierarchy including those that we consider in this paper.

Many applications of the 2D Toda hierarchy and its relatives have been found in mathematics and mathematical physics. In the 1990s, the 1D and 2D Toda hierarchies were applied to $2 \mathrm{D}$ gravity [25, 26, 27, 28] and $c=1$ string theory [29, 30, 31, 32, 33, 34] as well as mathematical aspects of random matrices and orthogonal polynomials [35, 36, 37]. This is also the place where the Ablowitz-Ladik hierarchy [38] (aka the relativistic Toda hierarchy [39]) plays a role. These studies also revealed new features of the $2 \mathrm{D}$ Toda hierarchy itself such as the Orlov-Schulman operators, additional symmetries and dispersionless analogues [40, 41]. Researches on the dispersionless 2D Toda hierarchy revived later on when a relation to interface dynamics and complex analysis was pointed out [42, 43].

Sources of new researches were discovered in the early 2000s in enumerative geometry of $\mathbb{C P}^{1}$ and $\mathbb{C}^{2}$ [44, 45, 46, 47, 48, 49, 50, 51, 52, and 4D $\mathcal{N}=2$ supersymmetric gauge theories [53, 54, 55, 56]. For example, a generating function of the double Hurwitz numbers of $\mathbb{C P}^{1}$ was shown to be a tau function of the 2D Toda hierarchy [45]. This tau function falls into a class of special tau functions called "hypergeometric tau functions" that was introduced around 2000 in a quite different context [57, 58, 59]. Intersection numbers of the Hilbert scheme of points on $\mathbb{C}^{2}$, too, give a hypergeometric tau function [50, 51]. On the other hand, Gromov-Witten invariants of $\mathbb{C P}^{1}$ yield a different kind of tau functions [47, 48].

This paper reviews our work in the last ten years on integrable structures of the melting crystal models [60, 61, 62, 63, 64]. We focus on two typical cases among these models of statistical mechanics. The first case is a statistical model of random 3D Young diagrams [65] (hence referred to as a "crystal 
model"). Its partition function may be also thought of as the simplest instanton partition function of 5D supersymmetric gauge theories [66]. The second case is a slight modification of the first case, and related to enumerative geometry [67] and topological string theory [68] of a Calabi-Yau threefold called the "resolved conifold". Our work have proved that the 1D Toda hierarchy and the Ablowitz-Ladik hierarchy underlie these two melting crystal models. Let us mention that such a relation between the resolved conifold and the Ablowitz-Ladik hierarchy was pointed out first by Brini [69]. It is remarkable that these two integrable hierarchies, both of which are reductions of the $2 \mathrm{D}$ Toda hierarchy [15, 36, 70], are also known to be the integrable structures of two typical random matrix models, namely the Hermitian and unitary random matrix models [25, 26, 27, 28, 35, 36, 37]. Technical clues of our work are the quantum torus algebra in the fermionic formalism, special algebraic relations in this algebra referred to as "shift symmetries", and the matrix factorization problem in the Lax formalism.

This paper is organized as follows. Section 2 is a review of the 2D Toda hierarchy formulated in the Lax and bilinear forms. Fundamental building blocks of the 2D Toda hierarchy such as the Lax operators, the dressing operators, the wave functions and the tau function are introduced along with various equations. The $1 \mathrm{D}$ Toda and Ablowitz-Ladik hierarchies are shown to be reductions of the 2D Toda hierarchy. The matrix factorization problem is also commented. Section 3 is a review of the fermionic formalism of the $2 \mathrm{D}$ Toda hierarchy. The fermionic formula of the tau function and its relation to the matrix factorization problem are explained. Relevant combinatorial notions such as partitions, Young diagrams and the Schur functions are also introduced here. The fermionic formula is illustrated for hypergeometric tau functions, in particular, the generating function of the double Hurwitz numbers. Sections 4 and 5 are devoted to the melting crystal models. In Section 4, the two melting crystal models are introduced. The partition functions are defined as sums of the Boltzmann weights over the set of all partitions. Fermionic expressions of these partition functions are also derived. In Section 5, integrable structures of the two melting crystal models are identified. The quantum torus algebra and its shift symmetries are reviewed. With the aid of these algebraic tools, the partition functions are converted to tau functions of the 2D Toda hierarchy. The first model thus turns out to be related to the 1D Toda hierarchy. The second model is further examined in the Lax formalism, and shown to be related to the Ablowitz-Ladik hierarchy. Section 6 concludes these reviews. 


\section{2D Toda hierarchy}

\subsection{Difference operators and infinite matrices}

The Lax formalism of the 2D Toda hierarchy is formulated by difference operators in the lattice coordinate $s$ [15]. These operators are linear combinations of the shift operators $e^{n \partial_{s}}$ (symbolically expressed as the exponential of $\left.\partial_{s}=\partial / \partial s\right)$ that act on functions of $s$ as $e^{n \partial_{s}} f(s)=f(s+n)$. A genuine difference operators is a finite linear combination

$$
A=\sum_{n=M}^{N} a_{n}(s) e^{n \partial_{s}} \quad \text { (operator of }[M, N] \text {-type) }
$$

of the shift operators. To formulate the 2D Toda hierarchy, we further use semi-infinite linear combinations of the form

$$
A=\sum_{n=-\infty}^{N} a_{n}(s) e^{n \partial_{s}} \quad \text { (operator of }(-\infty, N] \text { type) }
$$

and

$$
A=\sum_{n=M}^{\infty} a_{n}(s) e^{n \partial_{s}} \quad \text { (operator of }[M, \infty) \text { type). }
$$

These "pseudo-difference operators" are analogues of pseudo-differential operators in the Lax formalism of the KP hierarchy [16, 17]. Let ()$_{\geq 0}$ and $(\quad)_{<0}$ denote the projection

$$
(A)_{\geq 0}=\sum_{n \geq 0} a_{n}(s) e^{n \partial_{s}}, \quad(A)_{<0}=\sum_{n<0} a_{n}(s) e^{n \partial_{s}}
$$

to the $[0, \infty)$ and $(-\infty,-1]$ parts.

These difference operators are also represented by $\mathbb{Z} \times \mathbb{Z}$ matrices. The shift operators $e^{n \partial_{s}}$ correspond to the shift matrices

$$
\Lambda^{n}=\left(\delta_{i, j-n}\right)_{i, j \in \mathbb{Z}} .
$$

The multiplication operators $a(s)$ are represented by the diagonal matrices

$$
\operatorname{diag}(a(s))=\left(a(i) \delta_{i j}\right)_{i, j \in \mathbb{Z}} \cdot
$$

Thus a general difference operator of the form

$$
A=A\left(s, e^{\partial_{s}}\right)=\sum_{n \in \mathbb{Z}} a_{n}(s) e^{n \partial_{s}}
$$


is represented by the infinite matrix

$$
A(\Delta, \Lambda)=\sum_{n \in \mathbb{Z}} \operatorname{diag}\left(a_{n}(s)\right) \Lambda^{n}=\sum_{n \in \mathbb{Z}}\left(a_{n}(i) \delta_{i, j-n}\right)_{i, j \in \mathbb{Z}} .
$$

The shift operator $e^{\partial_{s}}$ and the multiplication operator $s$ satisfy the twisted canonical commutation relation

$$
\left[e^{\partial_{s}}, s\right]=e^{\partial_{s}} .
$$

This commutation relation can be translated to the language of matrices as

$$
[\Lambda, \Delta]=\Lambda
$$

where $\Delta$ denotes the the diagonal matrix

$$
\Delta=\operatorname{diag}(s)=\left(i \delta_{i j}\right)_{i, j \in \mathbb{Z}}
$$

that represents the multiplication operator $s$.

\subsection{Lax and Zakharov-Shabat equations}

The Lax formalism of the 2D Toda hierarchy uses two Lax operators $L, \bar{L} 1$ of type $(-\infty, 1]$ and $[1, \infty)$. From the point of view of symmetry, it is better to consider $L$ and $\bar{L}^{-1}$ rather than $L$ and $\bar{L}$. These operators admit freedom of gauge transformations $L \rightarrow e^{-f} \cdot L \cdot e^{f}, \bar{L} \rightarrow e^{-f} \cdot \bar{L} \cdot e^{f}$. We mostly use the gauge in which the leading coefficient of $L$ is equal to 1 :

$$
\begin{aligned}
L & =e^{\partial_{s}}+\sum_{n=1}^{\infty} u_{n} e^{(1-n) \partial_{s}}, \\
\bar{L}^{-1} & =\bar{u}_{0} e^{-\partial_{s}}+\sum_{n=1}^{\infty} \bar{u}_{n} e^{(n-1) \partial_{s}} .
\end{aligned}
$$

The coefficients $u_{n}$ and $\bar{u}_{n}$ are functions $u_{n}(s, \boldsymbol{t}, \overline{\boldsymbol{t}})$ and $\bar{u}_{n}(s, \boldsymbol{t}, \overline{\boldsymbol{t}})$ of $s$ and the time variables $\boldsymbol{t}, \overline{\boldsymbol{t}}$. To simplify notations, however, we shall frequently suppress $\boldsymbol{t}$ and $\overline{\boldsymbol{t}}$ as $u_{n}=u_{n}(s)$ and $\bar{u}_{n}=\bar{u}_{n}(s)$.

$L$ and $\bar{L}$ satisfy the Lax equations

$$
\begin{array}{ll}
\frac{\partial L}{\partial t_{n}}=\left[B_{n}, L\right], & \frac{\partial L}{\partial \bar{t}_{n}}=\left[\bar{B}_{n}, L\right], \\
\frac{\partial \bar{L}}{\partial t_{n}}=\left[B_{n}, \bar{L}\right], & \frac{\partial \bar{L}}{\partial \bar{t}_{n}}=\left[\bar{B}_{n}, \bar{L}\right],
\end{array}
$$

\footnotetext{
${ }^{1}$ In the earliest work [15, these Lax operators were denoted by $L, M$. These notations have been changed to $L, \bar{L}$ so as to use $M$ for the Orlov-Schulman operators. Also note that the bar ${ }^{-}$of $\bar{L}, \bar{t}_{k}, \bar{u}_{n}$, etc. does not mean complex conjugation.
} 
where $B_{n}$ and $\bar{B}_{n}$ are defined as

$$
B_{n}=\left(L^{n}\right)_{\geq 0}, \quad \bar{B}_{n}=\left(\bar{L}^{-n}\right)_{<0} .
$$

$B_{n}$ and $\bar{B}_{n}$, in turn, satisfy the Zakharov-Shabat equations

$$
\begin{aligned}
& \frac{\partial B_{n}}{\partial t_{m}}-\frac{\partial B_{m}}{\partial t_{n}}+\left[B_{m}, B_{n}\right]=0, \\
& \frac{\partial \bar{B}_{n}}{\partial \bar{t}_{m}}-\frac{\partial \bar{B}_{m}}{\partial \bar{t}_{n}}+\left[\bar{B}_{m}, \bar{B}_{n}\right]=0, \\
& \frac{\partial \bar{B}_{n}}{\partial t_{m}}-\frac{\partial B_{m}}{\partial \bar{t}_{n}}+\left[B_{m}, \bar{B}_{n}\right]=0 .
\end{aligned}
$$

Actually, the Lax equations and the Zakharov-Shabat equations are equivalent [15.

Since

$$
B_{1}=e^{\partial_{s}}+u_{1}, \quad \bar{B}_{1}=\bar{u}_{0} e^{-\partial_{s}},
$$

the lowest $(m=n=1)$ member of the third set of the Zakharov-Shabat equation reduces to the equations

$$
\begin{gathered}
\frac{\partial u_{1}(s)}{\partial \bar{t}_{1}}+\bar{u}_{0}(s+1)-\bar{u}_{0}(s)=0 \\
-\frac{\partial \bar{u}(s)_{0}}{\partial t_{1}}+\bar{u}_{0}(s)\left(u_{1}(s)-u_{1}(s-1)\right)=0 .
\end{gathered}
$$

Upon parametrizing $u_{1}$ and $\bar{u}_{0}$ with new dependent variable $\phi(s)=\phi(s, \boldsymbol{t}, \overline{\boldsymbol{t}})$ as

$$
u_{1}(s)=\frac{\partial \phi(s)}{\partial t_{1}}, \quad \bar{u}_{0}(s)=e^{\phi(s)-\phi(s-1)}
$$

these equations yields the 2D Toda field equation

$$
\frac{\partial^{2} \phi(s)}{\partial t_{1} \partial \bar{t}_{1}}+e^{\phi(s+1)-\phi(s)}-e^{\phi(s)-\phi(s-1)}=0 .
$$

\subsection{Dressing operators and wave functions}

The Lax operators $L, \bar{L}$ can be converted to the undressed form $e^{\partial_{s}}$ as

$$
L=W e^{\partial_{s}} W^{-1}, \quad \bar{L}=\bar{W} e^{\partial_{s}} \bar{W}^{-1}
$$

by dressing operators of the form

$$
W=1+\sum_{n=1}^{\infty} w_{n} e^{-n \partial_{s}}, \quad \bar{W}=\sum_{n=0}^{\infty} \bar{w}_{n} e^{n \partial_{s}}, \quad \bar{w}_{0} \neq 0 .
$$


One can further choose $W, \bar{W}$ to satisfy the Sato equations

$$
\begin{gathered}
\frac{\partial W}{\partial t_{k}}=B_{k} W-W e^{k \partial_{s}}, \quad \frac{\partial W}{\partial \bar{t}_{k}}=\bar{B}_{k} W \\
\frac{\partial \bar{W}}{\partial t_{k}}=B_{k} \bar{W}, \quad \frac{\partial \bar{W}}{\partial \bar{t}_{k}}=\bar{B}_{k} \bar{W}-W e^{-k \partial_{s}} .
\end{gathered}
$$

Upon substituting the expression

$$
B_{k}=\left(W e^{k \partial_{s}} W^{-1}\right)_{\geq 0}, \quad \bar{B}_{k}=\left(\bar{W} e^{-k \partial_{s}} \bar{W}^{-1}\right)^{-1}
$$

for $B_{k}$ 's and $\bar{B}_{k}$ 's, the Sato equations (2.7) turn into the closed system of evolution equations

$$
\begin{gathered}
\frac{\partial W}{\partial t_{k}}=-\left(W e^{k \partial_{s}} W^{-1}\right)_{<0} W, \quad \frac{\partial W}{\partial \bar{t}_{k}}=\left(\bar{W} e^{-k \partial_{s}} \bar{W}\right)_{<0} W \\
\frac{\partial \bar{W}}{\partial t_{k}}=\left(W e^{k \partial_{s}} W^{-1}\right)_{\geq 0} \bar{W}, \quad \frac{\partial \bar{W}}{\partial \bar{t}_{k}}=-\left(\bar{W} e^{-k \partial_{s}} \bar{W}^{-1}\right)_{\geq 0} \bar{W}
\end{gathered}
$$

for $W$ and $\bar{W}$. These equations and may be thought of as yet another formulation of the 2D Toda hierarchy, from which the Lax equations (2.3) can be recovered through the relation (2.6).

The dressing operators can be used to define the wave functions

$$
\Psi=\left(1+\sum_{k=1}^{\infty} w_{k} z^{-k}\right) z^{s} e^{\xi(\boldsymbol{t}, z)}, \quad \bar{\Psi}=\left(\sum_{k=0}^{\infty} \bar{w}_{k} z^{k}\right) z^{s} e^{\xi\left(\overline{\boldsymbol{t}}, z^{-1}\right)}
$$

where

$$
\xi(\boldsymbol{t}, z)=\sum_{k=1}^{\infty} t_{k} z^{k}, \quad \xi\left(\overline{\boldsymbol{t}}, z^{-1}\right)=\sum_{k=1}^{\infty} \bar{t}_{k} z^{-k} .
$$

The wave functions satisfy the auxiliary linear equations

$$
L \Psi=z \Psi, \quad \bar{L} \bar{\Psi}=z \bar{\Psi}
$$

and

$$
\begin{array}{ll}
\frac{\partial \Psi}{\partial t_{k}}=B_{k} \Psi, & \frac{\partial \Psi}{\partial \bar{t}_{k}}=\bar{B}_{k} \Psi \\
\frac{\partial \bar{\Psi}}{\partial t_{k}}=B_{k} \bar{\Psi}, & \frac{\partial \bar{\Psi}}{\partial \bar{t}_{k}}=\bar{B}_{k} \bar{\Psi}
\end{array}
$$




\subsection{Tau functions and bilinear equations}

The tau function $\tau=\tau(s, \boldsymbol{t}, \overline{\boldsymbol{t}})$ of the $2 \mathrm{D}$ Toda hierarchy is related to the wave functions as 2

$$
\begin{aligned}
& \Psi(s, \boldsymbol{t}, \overline{\boldsymbol{t}}, z)=\frac{\tau\left(s-1, \boldsymbol{t}-\left[z^{-1}\right], \overline{\boldsymbol{t}}\right)}{\tau(s-1, \boldsymbol{t}, \overline{\boldsymbol{t}})} z^{s} e^{\xi(\boldsymbol{t}, z)} \\
& \bar{\Psi}(s-1, \boldsymbol{t}, \overline{\boldsymbol{t}}, z)=\frac{\tau(s, \boldsymbol{t}, \overline{\boldsymbol{t}}-[z])}{\tau(s-1, \boldsymbol{t}, \overline{\boldsymbol{t}})} z^{s} e^{\xi\left(\overline{\boldsymbol{t}}, z^{-1}\right)}
\end{aligned}
$$

where

$$
[z]=\left(z, z^{2} / 2, \cdots, z^{k} / k, \cdots\right) .
$$

Given the pair $\Psi, \bar{\Psi}$ of wave functions, one can define the tau function as a kind of potential that satisfy these relations.

The tau function satisfies an infinite number of Hirota equations. The first three members of these Hirota equations read

$$
\begin{gathered}
D_{1} \bar{D}_{1} \tau(s, \boldsymbol{t}, \overline{\boldsymbol{t}}) \cdot \tau(s, \boldsymbol{t}, \overline{\boldsymbol{t}})+2 \tau(s+1, \boldsymbol{t}, \overline{\boldsymbol{t}}) \tau(s-1, \boldsymbol{t}, \overline{\boldsymbol{t}})=0, \\
\left(D_{2}+D_{1}^{2}\right) \tau(s+1, \boldsymbol{t}, \overline{\boldsymbol{t}}) \cdot \tau(s, \boldsymbol{t}, \overline{\boldsymbol{t}})=0, \\
\left(\bar{D}_{2}+\bar{D}_{1}^{2}\right) \tau(s, \boldsymbol{t}, \overline{\boldsymbol{t}}) \cdot \tau(s+1, \boldsymbol{t}, \overline{\boldsymbol{t}})=0,
\end{gathered}
$$

where we have used Hirota's notation

$$
\begin{aligned}
& P\left(D_{1}, D_{2}, \ldots, \bar{D}_{1}, \bar{D}_{2}, \ldots\right) f(\boldsymbol{t}, \overline{\boldsymbol{t}}) \cdot g(\boldsymbol{t}, \overline{\boldsymbol{t}}) \\
& \quad=\left.P\left(\partial_{1}^{\prime}-\partial_{1}, \partial_{2}^{\prime}-\partial_{2}, \ldots, \bar{\partial}_{1}^{\prime}-\bar{\partial}_{1}, \bar{\partial}_{2}^{\prime}-\bar{\partial}_{2}, \ldots\right) f\left(\boldsymbol{t}^{\prime}, \overline{\boldsymbol{t}}^{\prime}\right) g(\boldsymbol{t}, \overline{\boldsymbol{t}})\right|_{\boldsymbol{t}^{\prime}=\boldsymbol{t}},
\end{aligned}
$$

where $\partial_{k}, \partial_{k}^{\prime}, \bar{\partial}_{k}, \bar{\partial}_{k}^{\prime}$ denote the derivatives $\partial_{k}=\partial / \partial t_{k}, \partial_{k}^{\prime}=\partial / \partial t_{k}^{\prime}, \bar{\partial}_{k}=$ $\partial / \partial \bar{t}_{k}, \bar{\partial}_{k}^{\prime}=\partial / \partial \bar{t}_{k}^{\prime}$. The first equation of (2.13) amounts to the 2D Toda field equation (2.5). The infinite system of Hirota equations can be encoded to (and decoded from) the single bilinear equation

$$
\begin{aligned}
& \oint \frac{d z}{2 \pi i} z^{s^{\prime}-s} e^{\xi\left(\boldsymbol{t}^{\prime}-\boldsymbol{t}, z\right)} \tau\left(s^{\prime}, \boldsymbol{t}^{\prime}-\left[z^{-1}\right], \overline{\boldsymbol{t}}^{\prime}\right) \tau\left(s, \boldsymbol{t}+\left[z^{-1}\right], \overline{\boldsymbol{t}}\right) \\
& \quad=\oint \frac{d z}{2 \pi i} z^{s^{\prime}-s} e^{\xi\left(\overline{\boldsymbol{t}}^{\prime}-\overline{\boldsymbol{t}}, z^{-1}\right)} \tau\left(s^{\prime}+1, \boldsymbol{t}^{\prime}, \overline{\boldsymbol{t}}^{\prime}-[z]\right) \tau(s-1, \boldsymbol{t}, \overline{\boldsymbol{t}}+[z]),
\end{aligned}
$$

where the symbol $\oint$ means extracting the "residue" of a (formal) Laurent series:

$$
\oint \sum_{n=-\infty}^{\infty} \frac{d z}{2 \pi i} a_{n} z^{n}=a_{-1} .
$$

\footnotetext{
${ }^{2}$ These relations differ from those commonly used in the literature. We have replaced $\tau(s, \boldsymbol{t}, \overline{\boldsymbol{t}})$ therein by $\tau(s-1, \boldsymbol{t}, \overline{\boldsymbol{t}})$ so as to consistent with the convention of our fermionic formalism.
} 
Analytically, this symbol on the left side of the equation is understood to be the contour integral along a sufficiently large circle $|z|=R$, and that of the right side is the contour integral along a sufficiently small circle $|z|=R^{-1}$.

Various bilinear equations for the tau function can be derived from (2.14) by specialization of $\boldsymbol{t}^{\prime}, \overline{\boldsymbol{t}}^{\prime}$ and $s^{\prime}$. The Hirota equations (2.13) are obtained by Taylor expansion of (2.14) at $\boldsymbol{t}^{\prime}=\boldsymbol{t}$ and $\overline{\boldsymbol{t}}^{\prime}=\overline{\boldsymbol{t}}$ to low orders upon letting $s^{\prime}=s, s \pm 1$. More systematic derivation of Hirota equations uses the polynomials $S_{n}(\boldsymbol{t}), n=0,1, \ldots$, defined by the generating function

$$
\sum_{n=0}^{\infty} S_{n}(\boldsymbol{t}) z^{n}=\exp \left(\sum_{k=1}^{\infty} t_{k} z^{k}\right) \text {. }
$$

These polynomials are building blocks the Schur functions as well (we refer to Macdonald's book [71] for the notions of the Schur functions, partitions and Young diagrams). Thus a complete set of Hirota equations can be obtained in the generating functional form

$$
\begin{aligned}
& \sum_{n=0}^{\infty} S_{n}(-2 \boldsymbol{a}) S_{n+s^{\prime}-s+1}\left(\tilde{D}_{\boldsymbol{t}}\right) e^{\left\langle\boldsymbol{a}, D_{\boldsymbol{t}}\right\rangle+\left\langle\overline{\boldsymbol{a}}, D_{\overline{\boldsymbol{t}}}\right\rangle} \tau(s, \boldsymbol{t}, \overline{\boldsymbol{t}}) \cdot \tau\left(s^{\prime}, \boldsymbol{t}, \overline{\boldsymbol{t}}\right) \\
& \quad=\sum_{n=0}^{\infty} S_{n}(-2 \overline{\boldsymbol{a}}) S_{n-s^{\prime}+s-1}\left(\tilde{D}_{\overline{\boldsymbol{t}}}\right) e^{\left\langle\boldsymbol{a}, D_{\boldsymbol{t}}\right\rangle+\left\langle\overline{\boldsymbol{a}}, D_{\overline{\boldsymbol{t}}}\right\rangle} \tau(s-1, \boldsymbol{t}, \overline{\boldsymbol{t}}) \cdot \tau\left(s^{\prime}+1, \boldsymbol{t}, \overline{\boldsymbol{t}}\right),
\end{aligned}
$$

where $\boldsymbol{a}=\left(a_{1}, a_{2}, \ldots\right)$ and $\overline{\boldsymbol{a}}=\left(\bar{a}_{1}, \bar{a}_{2}, \ldots\right)$ are auxiliary variables, $\left\langle\boldsymbol{a}, D_{\boldsymbol{t}}\right\rangle$ and $\left\langle\overline{\boldsymbol{a}}, D_{\overline{\boldsymbol{t}}}\right\rangle$ are the linear combinations

$$
\left\langle\boldsymbol{a}, D_{\boldsymbol{t}}\right\rangle=\sum_{k=1}^{\infty} a_{k} D_{k}, \quad\left\langle\overline{\boldsymbol{a}}, \bar{D}_{\overline{\boldsymbol{t}}}\right\rangle=\sum_{k=1}^{\infty} \bar{a}_{k} \bar{D}_{k}
$$

of $D_{k}$ 's and $\bar{D}_{k}$ 's, and $S_{n}\left(\tilde{D}_{\boldsymbol{t}}\right)$ and $S_{n}\left(\tilde{D}_{\overline{\boldsymbol{t}}}\right)$ are defined by substituting the variables $\boldsymbol{t}$ of $S_{n}(\boldsymbol{t})$ for the Hirota bilinear operators

$$
\tilde{D}_{\boldsymbol{t}}=\left(D_{1}, D_{2} / 2, \ldots, D_{n} / k, \ldots\right), \quad \tilde{D}_{\overline{\boldsymbol{t}}}=\left(\bar{D}_{1}, \bar{D}_{2} / 2, \ldots, \bar{D}_{k} / k, \ldots\right) .
$$

\subsection{Orlov-Schulman operators}

Following the idea of Orlov and Schulman [72], one can introduce a Toda version of the Orlov-Schulman operator of the KP hierarchy. Actually, we 
need two Orlov-Schulman operators of the form

$$
\begin{gathered}
M=\sum_{k=1}^{\infty} k t_{k} L^{k}+s+\sum_{n=1}^{\infty} v_{n} L^{-n}, \\
\bar{M}=-\sum_{k=1}^{\infty} k \bar{t}_{k} \bar{L}^{-k}+s+\sum_{n=1}^{\infty} \bar{v}_{n} \bar{L}^{n}
\end{gathered}
$$

where $v_{n}$ and $\bar{v}_{n}$ are new dependent variables. These operators are defined in terms of the dressing operators as

$$
\begin{aligned}
& M=W\left(s+\sum_{k=1}^{\infty} k t_{k} e^{k \partial_{s}}\right) W^{-1}, \\
& \bar{M}=\bar{W}\left(s-\sum_{k=1}^{\infty} k \bar{t}_{k} e^{-k \partial_{s}}\right) \bar{W}^{-1}
\end{aligned}
$$

and satisfy the Lax equations

$$
\begin{array}{ll}
\frac{\partial M}{\partial t_{n}}=\left[B_{n}, M\right], & \frac{\partial M}{\partial \bar{t}_{n}}=\left[\bar{B}_{n}, M\right], \\
\frac{\partial \bar{M}}{\partial t_{n}}=\left[B_{n}, \bar{M}\right], & \frac{\partial \bar{M}}{\partial \bar{t}_{n}}=\left[\bar{B}_{n}, \bar{M}\right]
\end{array}
$$

of the same form as the Lax equations (2.3) for $L, \bar{L}$. Moreover, the twisted canonical commutation relations

$$
[L, M]=L, \quad[\bar{L}, \bar{M}]=\bar{L}
$$

are satisfied as a result of the commutation relation (2.1) of $e^{\partial_{s}}$ and $s$.

These equations form an extended Lax formalism of the 2D Toda hierarchy. One can thereby formulate additional symmetries of $W_{1+\infty}$ type [40, 41]. These additional symmetries play a central role in the so called "string equations" for various special solutions [29, 30, 31, 32, 33, 34, 73. Moreover, general solutions of the 2D Toda hierarchy, too, can be captured by the generalization

$$
L=f(\bar{L}, \bar{M}), \quad M=g(\bar{L}, \bar{M})
$$

of those string equations [41, 74]. 


\subsection{Two reductions of $2 \mathrm{D}$ Toda hierarchy}

\subsubsection{D Toda hierarchy}

The 1D Toda hierarchy is a reduction of the 2D Toda hierarchy in which all dynamical variables depend on the time variables $\boldsymbol{t}, \overline{\boldsymbol{t}}$ through the difference $\boldsymbol{t}-\overline{\boldsymbol{t}}$. In the Lax formalism, the 1D reduction can be achieved by imposing the condition 3

$$
L=\bar{L}^{-1}
$$

Both sides of this equation become a difference operator of the form

$$
\mathfrak{L}=e^{\partial_{s}}+b+c e^{-\partial_{s}}, \quad b=u_{1}, \quad c=\bar{u}_{0},
$$

which satisfies the Lax equations

$$
\frac{\partial \mathfrak{L}}{\partial t_{k}}=\left[B_{k}, \mathfrak{L}\right], \quad \frac{\partial \mathfrak{L}}{\partial \bar{t}_{k}}=\left[\bar{B}_{k}, \mathfrak{L}\right] .
$$

Since (2.21) implies that $B_{k}, \bar{B}_{k}$ and $\mathfrak{L}$ are linearly related as

$$
B_{k}+\bar{B}_{k}=\mathfrak{L}^{k}
$$

the time evolutions with respect to $\boldsymbol{t}$ and $\overline{\boldsymbol{t}}$ are also linearly related as

$$
\frac{\partial \mathfrak{L}}{\partial t_{k}}+\frac{\partial \mathfrak{L}}{\partial \bar{t}_{k}}=\left[B_{k}, \mathfrak{L}\right]+\left[\bar{B}_{k}, \mathfrak{L}\right]=0
$$

Thus the reduced system has just one set of independent Lax equations

$$
\frac{\partial \mathfrak{L}}{\partial t_{k}}=\left[B_{k}, \mathfrak{L}\right], \quad B_{k}=\left(\mathfrak{L}^{k}\right)_{\geq 0} .
$$

\subsubsection{Ablowitz-Ladik hierarchy}

The reduction to the Ablowitz-Ladik hierarchy is a kind of "rational reduction" [70]. This is achieved by assuming that $L$ and $\bar{L}^{-1}$ are quotients

$$
L=B C^{-1}, \quad \bar{L}^{-1}=C B^{-1}
$$

of two difference operators of the form

$$
B=e^{\partial_{s}}-b, \quad C=1-c e^{-\partial_{s}} .
$$

\footnotetext{
${ }^{3}$ In the earliest work [15], a condition of the form $L+L^{-1}=\bar{L}+\bar{L}^{-1}$ is proposed for the $1 \mathrm{D}$ reduction. This condition is related to the structure of soliton solutions of the Toda lattice [3, 6].
} 
$B^{-1}$ and $C^{-1}$ are understood to be difference operators of type $[0, \infty)$ and $(-\infty, 0]$. More explicitly,

$$
\begin{aligned}
& B^{-1}=-\sum_{k=0}^{\infty}\left(b^{-1} e^{\partial_{s}}\right)^{k} b^{-1}=-b(s)^{-1}-\sum_{k=1}^{\infty} b(s)^{-1} \cdots b(s+k)^{-1} e^{k \partial_{s}}, \\
& C^{-1}=1+\sum_{k=1}^{\infty}\left(c e^{-\partial_{s}}\right)^{k}=1+\sum_{k=1}^{\infty} c(s) c(s-1) \cdots c(s-k+1) e^{-k \partial_{s}}
\end{aligned}
$$

where $b(s)$ and $c(s)$ are abbreviations of $c(s, \boldsymbol{t}, \overline{\boldsymbol{t}})$ and $c(s, \boldsymbol{t}, \overline{\boldsymbol{t}})$. Under this interpretation, $C B^{-1}$ is not the inverse of $B C^{-1}$. Thus trivial situation where $L=\bar{L}=e^{\partial_{s}}$ can be avoided.

The Lax equations (2.3) of the 2D Toda hierarchy can be reduced to (and derived from) the equations

$$
\begin{aligned}
& \frac{\partial B}{\partial t_{k}}=\left(\left(B C^{-1}\right)^{k}\right)_{\geq 0} B-B\left(\left(C^{-1} B\right)^{k}\right)_{\geq 0}, \\
& \frac{\partial C}{\partial t_{k}}=\left(\left(B C^{-1}\right)^{k}\right)_{\geq 0} C-C\left(\left(C^{-1} B\right)^{k}\right)_{\geq 0}, \\
& \frac{\partial B}{\partial \bar{t}_{k}}=\left(\left(C B^{-1}\right)^{k}\right)_{<0} B-B\left(\left(B^{-1} C\right)^{k}\right)_{<0}, \\
& \frac{\partial C}{\partial \bar{t}_{k}}=\left(\left(C B^{-1}\right)^{k}\right)_{<0} C-C\left(\left(B^{-1} C\right)^{k}\right)_{<0} .
\end{aligned}
$$

Note that this is a closed system of evolution equations for $B$ and $C$. This implies that the reduced form (2.24) of $L$ and $\bar{L}^{-1}$ is preserved by the time evolutions of the 2D Toda hierarchy.

The reduction condition to the Ablowitz-Ladik hierarchy can be reformulated in the alternative form

$$
L=\tilde{C}^{-1} \tilde{B}, \quad \bar{L}^{-1}=\tilde{B}^{-1} \tilde{C},
$$

where $\tilde{B}$ and $\tilde{C}$ are difference operators of the form

$$
\tilde{B}=e^{\partial_{s}}-\tilde{b}, \quad \tilde{C}=1-\tilde{c} e^{-\partial_{s}} .
$$

Just like $B^{-1}$ and $C^{-1}$ in (2.24), $\tilde{B}^{-1}$ and $\tilde{C}^{-1}$ are understood to be difference operators of type $[0, \infty)$ and $(-\infty, 0]$. The Lax equations (2.3) of the $2 \mathrm{D}$ Toda 
hierarchy can be reduced to the equations

$$
\begin{aligned}
& \left.\frac{\partial \tilde{B}}{\partial t_{k}}=\left(\left(\tilde{B} \tilde{C}^{-1}\right)^{k}\right)_{\geq 0} \tilde{B}-\tilde{B}\left(\tilde{C}^{-1} \tilde{B}\right)^{k}\right)_{\geq 0}, \\
& \frac{\partial \tilde{C}}{\partial t_{k}}=\left(\left(\tilde{B} \tilde{C}^{-1}\right)^{k}\right)_{\geq 0} \tilde{C}-\tilde{C}\left(\left(\tilde{C}^{-1} \tilde{B}\right)^{k}\right)_{\geq 0}, \\
& \frac{\partial \tilde{B}}{\partial \bar{t}_{k}}=\left(\left(\tilde{C} \tilde{B}^{-1}\right)^{k}\right)_{<0} \tilde{B}-\tilde{B}\left(\left(\tilde{B}^{-1} \tilde{C}\right)^{k}\right)_{<0}, \\
& \frac{\partial \tilde{C}}{\partial \bar{t}_{k}}=\left(\left(\tilde{C} \tilde{B}^{-1}\right)^{k}\right)_{<0} \tilde{C}-\tilde{C}\left(\left(\tilde{B}^{-1} \tilde{C}\right)^{k}\right)_{<0}
\end{aligned}
$$

for these operators as well.

The second reduction condition $(2.26)$ is directly related to an auxiliary linear problem of the relativistic Toda hierarchy [39]. If the Lax operators are factorized in that form, the linear equations (2.10) for the wave functions can be converted to the "generalized eigenvalue problem"

$$
\tilde{B} \Psi=z \tilde{C} \Psi, \quad \tilde{B} \bar{\Psi}=z \tilde{C} \bar{\Psi} .
$$

A generalized eigenvalue problem of this form is used in Bruschi and Ragnisco's scalar-valued Lax formalism [75] of the relativistic Toda lattice. Moreover, as pointed out by Kharchev et al. [36], this generalized eigenvalue problem can be derived from the traditional $2 \times 2$ matrix-valued Lax formalism [38] of the Ablowitz-Ladik hierarchy.

\subsection{Matrix factorization problem}

General solutions of the 2D Toda hierarchy can be captured by a factorization problem [24] of the form

$$
\exp \left(\sum_{k=1}^{\infty} t_{k} \Lambda^{k}\right) U \exp \left(-\sum_{k=1}^{\infty} \bar{t}_{k} \Lambda^{-k}\right)=W^{-1} \bar{W}
$$

where $U$ is a given (invertible) constant $\mathbb{Z} \times \mathbb{Z}$ matrix. The problem is to find two $\mathbb{Z} \times \mathbb{Z}$ matrices $W=W(\boldsymbol{t}, \overline{\boldsymbol{t}})$ and $\bar{W}=\bar{W}(\boldsymbol{t}, \overline{\boldsymbol{t}})$ that are triangular matrices of the form

$$
W=1+\sum_{n=1}^{\infty} \operatorname{diag}\left(w_{n}(s)\right) \Lambda^{-n}, \quad \bar{W}=\sum_{n=0}^{\infty} \operatorname{diag}\left(\bar{w}_{n}(s)\right) \Lambda^{n}, \quad \bar{w}_{0} \neq 0 .
$$

Note that $W$ and $\bar{W}$ amount to the dressing operators of the last section by the correspondence

$$
A\left(s, e^{\partial_{s}}\right)=\sum_{n \in \mathbb{Z}} a_{n}(s) e^{n \partial_{s}} \longleftrightarrow A(\Delta, \Lambda)=\sum_{n \in \mathbb{Z}} \operatorname{diag}\left(a_{n}(s)\right)_{s \in \mathbb{Z}} \Lambda^{n}
$$


of difference operators and $\mathbb{Z} \times \mathbb{Z}$ matrices.

Since $W$ and $\bar{W}$ are lower and upper triangular matrices, the factorization problem (2.29) is an infinite dimensional analogue of the Gauss decomposition for finite matrices. If $W$ and $\bar{W}$ satisfy the factorization problem (2.29), one can readily derive the equations

$$
\begin{gathered}
\frac{\partial W}{\partial t_{k}} W^{-1}+W \Lambda^{k} W^{-1}=\frac{\partial \bar{W}}{\partial t_{k}} \bar{W}^{-1} \\
\frac{\partial W}{\partial \bar{t}_{k}} W^{-1}=\frac{\partial \bar{W}}{\partial \bar{t}_{k}} \bar{W}^{-1}+\bar{W} \Lambda^{-k} \bar{W}^{-1}
\end{gathered}
$$

Splitting these equations to the ()$_{\geq 0}$ and ()$_{<0}$ parts, one can see that these equations are equivalent to the Sato equations (2.8). Thus the factorization problem yields a solution of the 2D Toda hierarchy.

In analogy with the procedure of the Gauss decomposition for finite matrices, one can express the matrix elements of $W$ and $\bar{W}$ as quotients of semi-infinite minors of

$$
U(\boldsymbol{t}, \overline{\boldsymbol{t}})=\left(U_{i j}(\boldsymbol{t}, \overline{\boldsymbol{t}})\right)_{i, j \in \mathbb{Z}}=\exp \left(\sum_{k=1}^{\infty} t_{k} \Lambda^{k}\right) U \exp \left(-\sum_{k=1}^{\infty} \bar{t}_{k} \Lambda^{-k}\right) .
$$

The common denominator of these quotients is a principal minor of $U(\boldsymbol{t}, \overline{\boldsymbol{t}})$, and can be identified with the tau function: 4

$$
\tau(s, \boldsymbol{t}, \overline{\boldsymbol{t}})=\operatorname{det}\left(U_{i j}(\boldsymbol{t}, \overline{\boldsymbol{t}})\right)_{i, j \leq s} .
$$

The determinant expression of the matrix elements of $W$ and $\bar{W}$ reproduces the generating functional expression

$$
\begin{aligned}
1+\sum_{n=1}^{\infty} w_{n} z^{-n} & =\frac{\tau\left(s-1, \boldsymbol{t}-\left[z^{-1}\right], \boldsymbol{t}\right)}{\tau(s-1, \boldsymbol{t}, \overline{\boldsymbol{t}})}, \\
\sum_{n=0}^{\infty} \bar{w}_{n} z^{n} & =\frac{\tau(s, \boldsymbol{t}, \overline{\boldsymbol{t}}-[z])}{\tau(s-1, \boldsymbol{t}, \overline{\boldsymbol{t}})}
\end{aligned}
$$

of $w_{n}$ 's and $\bar{w}_{n}$ 's, which implies the relation (2.12).

These formal computations can be justified rigorously [24] in the case where $U$ is given by the quotient

$$
U=W_{0}^{-1} \bar{W}_{0}
$$

\footnotetext{
${ }^{4}$ This is a place where the aforementioned modification of the definition of $\tau(s, \boldsymbol{t}, \overline{\boldsymbol{t}})$ affects the outcome. In the earlier literature, the right hand side of this formula is the minor for $i, j<s$ rather than $i, j \leq s$.
} 
of two triangular matrices of the same form as $W$ and $\bar{W}$. In this case, $W_{0}$ and $\bar{W}_{0}$ can be identified with the initial values of $W$ and $\bar{W}$ :

$$
W_{0}=\left.W\right|_{\boldsymbol{t}=\overline{\boldsymbol{t}}=\mathbf{0}}, \quad \bar{W}_{0}=\left.\bar{W}\right|_{\boldsymbol{t}=\overline{\boldsymbol{t}}=\mathbf{0}} .
$$

In other words, the factorization problem (2.29) in this setup solves the initial value problem of the Sato equations (2.8).

The determinant formula (2.32) has many implications. First, this is an analogue of the determinant formula of the tau functions of the KP hierarchy. Since $U$ is an element of the "group" GL( $\infty)$ 5, it is GL( $\infty)$ itself that plays the role of the infinite-dimensional Grassmann manifold in the case of the KP hierarchy. More precisely, the true phase space lies in the product of two flag manifolds in which $W$ and $\bar{W}$ live. Second, the generating matrix $U$ is related to the generalized string equations (2.20). These equations are a consequence of the algebraic relations

$$
\Lambda U=U f(\Delta, \Lambda), \quad \Delta U=U g(\Delta, \Lambda)
$$

satisfied by $\Lambda, \Delta$ and $U$. Third, the determinant formula (2.32) can be translated to the language of a $2 \mathrm{D}$ complex free fermion system. Let us turn to this fermionic formalism of the $2 \mathrm{D}$ Toda hierarchy.

\section{Fermionic formalism}

\subsection{Complex free fermion system}

Let

$$
\psi(z)=\sum_{n \in \mathbb{Z}} \psi_{n} z^{-n-1}, \quad \psi^{*}(z)=\sum_{n \in \mathbb{Z}} \psi_{n}^{*} z^{-n}
$$

denote the conjugate pair of $2 \mathrm{D}$ complex free fermion fields. For convenience, we use integers rather than half-integers for the labels of Fourier modes $\psi_{n}, \psi_{n}^{*}$. The Fourier modes satisfy the anti-commutation relations

$$
\psi_{m} \psi_{n}^{*}+\psi_{n}^{*} \psi_{m}=\delta_{m+n, 0}, \quad \psi_{m} \psi_{n}+\psi_{n} \psi_{m}=0, \quad \psi_{m}^{*} \psi_{n}^{*}+\psi_{n}^{*} \psi_{m}^{*}=0 .
$$

$\psi_{i}$ 's and $\psi_{i}^{*}$ 's are understood to be linear operators on the fermionic Fock spaces. They act on the Fock space $\mathcal{H}$ from the left side and on its dual space $\mathcal{H}^{*}$ from the right side. These Fock spaces are decomposed to charge- $s$ sectors $\mathcal{H}_{s}, \mathcal{H}_{s}^{*}, s \in \mathbb{Z}$. Let $\langle s|$ and $|s\rangle$ denote the ground states in $\mathcal{H}_{s}$ and $\mathcal{H}_{s}^{*}: 6$

$$
\left\langle s\left|=\left\langle-\infty\left|\cdots \psi_{s-1}^{*} \psi_{s}^{*}, \quad\right| s\right\rangle=\psi_{-s} \psi_{-s+1} \cdots\right|-\infty\right\rangle .
$$

\footnotetext{
${ }^{5}$ This notation is used here in a loose sense and not intended to denote a true group.

${ }^{6}$ The shift of $s$ in (2.12) and (2.32) is related to this definition of the ground states.
} 
Excited states are labelled by partitions $\lambda=\left(\lambda_{1}, \lambda_{2}, \cdots, \lambda_{n}, 0,0, \cdots\right), \lambda_{1} \geq$ $\lambda_{2} \geq \cdots \geq 0$, of arbitrary length as

$$
\begin{gathered}
\langle\lambda, s|=\langle s| \psi_{-s} \cdots \psi_{n-1-s} \psi_{\lambda_{n}-n+1+s}^{*} \cdots \psi_{\lambda_{1}+s}^{*} \\
|\lambda, s\rangle=\psi_{-\lambda_{1}-s} \cdots \psi_{-\lambda_{n}+n-1-s} \psi_{-n+1+s}^{*} \cdots \psi_{s}^{*}|s\rangle
\end{gathered}
$$

$\langle s|$ and $|s\rangle$ are identified with $\langle\emptyset, s|$ and $|\emptyset, s\rangle .|\lambda, s\rangle$ and $\langle\lambda, s|$ represent a state in which the semi-infinite subset $\left\{\lambda_{i}-i+1+s\right\}_{i=1}^{\infty}$ of the set $\mathbb{Z}$ of all energy levels are occupied by particles. These vectors form dual bases of $\mathcal{H}_{s}$ and $\mathcal{H}_{s}^{*}$ :

$$
\langle\lambda, r \mid \mu, s\rangle=\delta_{\lambda \mu} \delta_{r s}
$$

The normal ordered fermion bilinears

$$
: \psi_{-i} \psi_{j}^{*}:=\psi_{-i} \psi_{j}^{*}-\left\langle 0\left|\psi_{-i} \psi_{j}^{*}\right| 0\right\rangle, \quad i, j \in \mathbb{Z}
$$

where

$$
\left\langle 0\left|\psi_{-i} \psi_{j}^{*}\right| 0\right\rangle= \begin{cases}1 & \text { if } i=j \leq 0 \\ 0 & \text { otherwise }\end{cases}
$$

span the one-dimensional central extension $\widehat{\mathrm{gl}}(\infty)$ of the Lie algebra $\mathrm{gl}(\infty)$ of $\mathbb{Z} \times \mathbb{Z}$ matrices [76, 77]. gl $(\infty)$ consists of infinite matrices $A=\left(a_{i j}\right)_{i, j \in \mathbb{Z}}$ that correspond to difference operators of finite type (i.e., of $[M, N]$-type for a pair of integers $M, N$ that can depend on $A$ ). For such a matrix $A \in \operatorname{gl}(\infty)$, the fermion bilinear

$$
\widehat{A}=\sum_{i, j \in \mathbb{Z}} a_{i j}: \psi_{-i} \psi_{j}^{*}:
$$

becomes a well-defined linear operator on the Fock space, and preserves the charge in the sense that

$$
\langle\lambda, r|\widehat{A}| \mu, s\rangle=0 \quad \text { if } r \neq s
$$

The elements of $\widehat{\mathrm{gl}}(\infty)$ satisfy the commutation relation

$$
[\widehat{A}, \widehat{B}]=\widehat{[A, B]}+\gamma(A, B)
$$

with the $c$-number cocycle

$$
\gamma(A, B)=\sum_{i>0, j \leq 0}\left(a_{i j} b_{j i}-b_{i j} a_{j i}\right)
$$




\subsection{Vertex operators and Schur functions}

We here introduce the special fermion bilinears

$$
J_{m}=\widehat{\Lambda^{m}}=\sum_{n \in \mathbb{Z}}: \psi_{m-n} \psi_{n}^{*}:, \quad m \in \mathbb{Z},
$$

which satisfy the commutation relations

$$
\left[J_{m}, J_{n}\right]=m \delta_{m+n}
$$

of the Heisenberg algebra. These operators are used to construct vertex operators. The matrix elements of such a vertex operator with respect to the vectors $\langle\lambda, s|$ and $|\mu, s\rangle$ are related to the Schur and skew Schur functions. Actually, there are two different types of vertex operators that correspond to different formulations of these functions.

Vertex operators of the first type are given by the product

$$
\Gamma_{ \pm}(\boldsymbol{x})=\prod_{i \geq 1} \Gamma_{ \pm}\left(x_{i}\right), \quad \boldsymbol{x}=\left(x_{1}, x_{2}, \ldots\right)
$$

of the elementary vertex operators

$$
\Gamma_{ \pm}(x)=\exp \left(\sum_{k=1}^{\infty} \frac{x^{k}}{k} J_{ \pm k}\right) .
$$

The matrix elements of these operators are the skew Schur functions $s_{\lambda / \mu}(\boldsymbol{x})$ in the sense of symmetric functions of $\boldsymbol{x}[65]$ :

$$
\left\langle\lambda, s\left|\Gamma_{-}(\boldsymbol{x})\right| \mu, s\right\rangle=\left\langle\mu, s\left|\Gamma_{+}(\boldsymbol{x})\right| \lambda, s\right\rangle=s_{\lambda / \mu}(\boldsymbol{x}) .
$$

In particular, if $\mu=\emptyset$, the matrix elements become the Schur functions $s_{\lambda}(\boldsymbol{x})$ :

$$
\left\langle\lambda, s\left|\Gamma_{-}(\boldsymbol{x})\right| s\right\rangle=\left\langle s\left|\Gamma_{+}(\boldsymbol{x})\right| \lambda, s\right\rangle=s_{\lambda}(\boldsymbol{x}) .
$$

Vertex operators of the second type are defined as

$$
\gamma_{ \pm}(\boldsymbol{t})=\exp \left(\sum_{k=1}^{\infty} t_{k} J_{ \pm k}\right)
$$

It is these operators $\gamma_{ \pm}(\boldsymbol{t})$ that are commonly used in the fermionic formula of tau functions of the KP and 2D Toda hierarchies [77, 23]. The matrix elements of $\gamma_{ \pm}(\boldsymbol{t})$ are the skew Schur functions $S_{\lambda / \mu}(\boldsymbol{t})$ of the $\boldsymbol{t}$-variables:

$$
\left\langle\lambda, s\left|\gamma_{-}(\boldsymbol{t})\right| \mu, s\right\rangle=\left\langle\mu, s\left|\gamma_{+}(\boldsymbol{t})\right| \lambda, s\right\rangle=S_{\lambda / \mu}(\boldsymbol{t})
$$


These functions are defined by the determinant formula

$$
S_{\lambda / \mu}(\boldsymbol{t})=\operatorname{det}\left(S_{\lambda_{i}-\mu_{j}-i+j}(\boldsymbol{t})\right)_{i, j=1}^{n}
$$

for partitions of the form $\lambda=\left(\lambda_{1}, \ldots, \lambda_{n}, 0,0, \ldots\right), \mu=\left(\mu_{1}, \ldots, \mu_{n}, 0,0, \ldots\right)$. $S_{n}(\boldsymbol{t})$ 's are the polynomials defined by the generating function (2.15).

$\gamma_{ \pm}(\boldsymbol{t})$ can be converted to $\Gamma_{ \pm}(\boldsymbol{x})$ by substituting

$$
t_{k}=\frac{1}{k} \sum_{i \geq 1} x_{i}^{k} .
$$

By the same transformation of variables, the polynomials $S_{n}(\boldsymbol{t})$ in $\boldsymbol{t}$ turn into the homogeneous symmetric function $h_{n}(\boldsymbol{x})$ of $\boldsymbol{x}$. The determinant formula (3.9) of $S_{\lambda / \mu}(\boldsymbol{t})$ thereby reproduces the Jacobi-Trudi formula of $s_{\lambda / \mu}(\boldsymbol{x})$.

\subsection{Fermionic formula of tau functions}

In terms of the foregoing vertex operators, the fermionic formula of Toda tau functions [21, 22, 23] reads 7

$$
\tau(s, \boldsymbol{t}, \overline{\boldsymbol{t}})=\left\langle s\left|\gamma_{+}(\boldsymbol{t}) g \gamma_{-}(-\overline{\boldsymbol{t}})\right| s\right\rangle,
$$

where $g$ is an element of the "group" $\widehat{\mathrm{GL}}(\infty) 8$ of Clifford operators (typically, the exponential $e^{\hat{A}}$ of a fermion bilinear $\left.\hat{A}\right)[76$, 77]. Such a Clifford operator induces a linear transformation on the linear span of $\psi_{i}$ 's and $\psi_{i}^{*}$ 's by the adjoint action:

$$
g \psi_{j} g^{-1}=\sum_{i \in \mathbb{Z}} \psi_{i} U_{i j}, \quad g \psi_{j}^{*} g^{-1}=\sum_{i \in \mathbb{Z}} \psi_{i}^{*} \tilde{U}_{i j}
$$

The coefficients $U_{i j}$ and $\tilde{U}_{i j}$ satisfy the orthogonality condition

$$
\sum_{k \in \mathbb{Z}} U_{i k} \tilde{U}_{j k}=\sum_{k \in \mathbb{Z}} U_{k i} \tilde{U}_{k j}=\delta_{i j}
$$

The fermionic formula (3.11) corresponds to the determinant formula (2.32) of the factorization problem (2.29) for the matrix $U=\left(U_{i j}\right)_{i, j \in \mathbb{Z}}$.

An immediate consequence of (3.11) is the Schur function expansion

$$
\tau(s, \boldsymbol{t}, \overline{\boldsymbol{t}})=\sum_{\lambda, \mu \in \mathcal{P}}\langle\lambda, s|g| \mu, s\rangle S_{\lambda}(\boldsymbol{t}) S_{\mu}(-\overline{\boldsymbol{t}}),
$$

\footnotetext{
${ }^{7}$ A prototype of this formula can be found in the work of Jimbo and Miwa [18.

${ }^{8}$ This notation, too, is used here in a loose sense just like $\mathrm{GL}(\infty)$.
} 
where $\mathcal{P}$ denotes the set of all partitions. This expansion is obtained by inserting the partition of unity

$$
1=\sum_{\lambda \in \mathcal{P}, s \in \mathbb{Z}}|\lambda, s\rangle\langle\lambda, s|
$$

to the two places among $\gamma_{+}(\boldsymbol{t}), g$ and $\gamma_{-}(-\overline{\boldsymbol{t}})$. This amounts to applying the Cauchy-Binet formula to the determinant formula (2.32). The three factors $\langle\lambda, s|g| \mu, s\rangle, S_{\lambda}(\boldsymbol{t}), S_{\mu}(-\overline{\boldsymbol{t}})$ may be thought of as minors of the three matrices on the right side of (2.31). As regards $S_{\lambda}(\boldsymbol{t})$ and $S_{\mu}(-\overline{\boldsymbol{t}})$, this is indeed a consequence of the special case

$$
S_{\lambda}(\boldsymbol{t})=\operatorname{det}\left(S_{\lambda_{i}-i+j}(\boldsymbol{t})\right)_{i, j=1}^{n}
$$

of the determinant formula (3.9) .

\subsection{Hypergeometric tau functions}

Let us illustrate the fermionic formula (3.11) in the case of hypergeometric tau functions [57, 58, 59]. This is the case where the generating operator $g$ of the tau function (3.11) corresponds to a diagonal matrix in $\mathrm{GL}(\infty)$.

Let $U=\left(e^{T_{i}} \delta_{i j}\right)_{i, j \in \mathbb{Z}}$ be such a diagonal matrix. The associated generating operator can be expressed as

$$
g=\exp \left(\sum_{n \in \mathbb{Z}} T_{n}: \psi_{-n} \psi_{n}^{*}:\right) .
$$

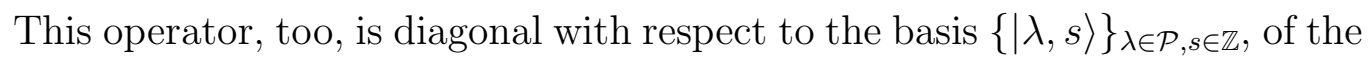
Fock space. Thus the tau function becomes a single sum over all partitions:

$$
\tau(s, \boldsymbol{t}, \overline{\boldsymbol{t}})=\sum_{\lambda \in \mathcal{P}}\langle\lambda, s|g| \lambda, s\rangle S_{\lambda}(\boldsymbol{t}) S_{\lambda}(-\overline{\boldsymbol{t}})
$$

The diagonal elements of $g$ takes the so called "contents product" form:

$$
\langle\lambda, s|g| \lambda, s\rangle=\langle s|g| s\rangle \prod_{(i, j) \in \lambda} r_{j-i+1+s}
$$

where $(i, j) \in \lambda$ means that $(i, j)$ runs over the cells of the Young diagram of shape $\lambda$, and $r_{n}$ 's are defined as

$$
r_{n}=e^{T_{n}-T_{n-1}}
$$


The $\lambda$-independent factor $\langle s|g| s\rangle$ can be expressed as

$$
\langle s|g| s\rangle=\frac{\prod_{i=1}^{\infty} e^{T_{-i+1+s}}}{\prod_{i=1}^{\infty} e^{T_{-i+1}}}= \begin{cases}\prod_{i=1}^{s} e^{T_{-i+1+s}} & \text { if } s>0 \\ 1 & \text { if } s=0 \\ \prod_{i=1}^{-s} e^{-T_{-i+1}} & \text { if } s<0\end{cases}
$$

These tau functions are called "hypergeometric" after the work of Orlov and Scherbin [57, 58, 59], because their work aimed at applications to multivariate hypergeometric functions. Actually, specialization of the parameters $\left\{T_{n}\right\}_{n \in \mathbb{Z}}$ yields a variety of examples other than hypergeometric functions. Earliest examples of these tau functions can be found in the studies of random matrix models [78, 79, 80, 81] and $c=1$ string theory [29, 33, 34]. Another source of examples is enumerative geometry of $\mathbb{C P}^{1}$ and $\mathbb{C}^{2}$ [45, 50, 51]. Recent researches of this class of tau functions are focussed on the double Hurwitz numbers [73, 82, 83, 84] and their variants [85, 86, 87, 88].

Let us briefly recall the tau function of the double Hurwitz numbers [45]. The generating operator takes such a form as

$$
g=Q^{L_{0}} e^{\beta K / 2}
$$

where $Q$ and $\beta$ are constants, and $L_{0}$ and $K$ are the special fermion bilinears

$$
\begin{gathered}
L_{0}=\widehat{\Delta}=\sum_{n \in \mathbb{Z}} n: \psi_{-n} \psi_{n}^{*}: \\
K=(\widehat{\Delta-1 / 2})^{2}=\sum_{n \in \mathbb{Z}}(n-1 / 2)^{2}: \psi_{-n} \psi_{n}^{*}:
\end{gathered}
$$

The diagonal matrix elements of these fermion bilinears can be computed as follows:

$$
\begin{gathered}
\left\langle\lambda, s\left|L_{0}\right| \lambda, s\right\rangle=|\lambda|+\frac{s(s+1)}{2}, \\
\langle\lambda, s|K| \lambda, s\rangle=\kappa(\lambda)+2 s|\lambda|+\frac{4 s^{3}-s}{12},
\end{gathered}
$$

where

$$
|\lambda|=\sum_{i=1}^{\infty} \lambda_{i}, \quad \kappa(\lambda)=\sum_{i=1}^{\infty} \lambda_{i}\left(\lambda_{i}-2 i+1\right) .
$$

This implies that

$$
\begin{gathered}
\left\langle\lambda, s\left|Q^{L_{0}}\right| \lambda, s\right\rangle=Q^{|\lambda|+s(s+1) / 2}, \\
\left\langle\lambda, s\left|e^{\beta K / 2}\right| \lambda, s\right\rangle=e^{\beta\left(\kappa(\lambda) / 2+s|\lambda|+\left(4 s^{3}-s\right) / 24\right)},
\end{gathered}
$$


Consequently, the tau function has a Schur function expansion of the form

$$
\tau(s, \boldsymbol{t}, \overline{\boldsymbol{t}})=\sum_{\lambda \in \mathcal{P}} Q^{|\lambda|+s(s+1) / 2} e^{\beta\left(\kappa(\lambda) / 2+s|\lambda|+\left(4 s^{3}-s\right) / 24\right)} S_{\lambda}(\boldsymbol{t}) S_{\lambda}(-\overline{\boldsymbol{t}}) .
$$

Its specialization

$$
\tau(0, \boldsymbol{t}, \overline{\boldsymbol{t}})=\sum_{\lambda \in \mathcal{P}} Q^{|\lambda|} e^{\beta \kappa(\lambda) / 2} S_{\lambda}(\boldsymbol{t}) S_{\lambda}(-\overline{\boldsymbol{t}})
$$

to $s=0$ is a genuine generating function of the double Hurwitz numbers.

Further specialization to $\overline{\boldsymbol{t}}=(-1,0,0, \ldots)$ becomes a generating function of the single Hurwitz numbers. The special value of the second Schur function at this point can be computed by the combinatorial formula [71]

$$
S_{\lambda}(1,0,0, \ldots)=\frac{\operatorname{dim} \lambda}{|\lambda| !}=\prod_{(i, j) \in \lambda} h(i, j)^{-1},
$$

where $h(i, j)$ is the hook length of the cell $(i, j)$ in the Young diagram of shape $\lambda$, and $\operatorname{dim} \lambda$ is the number of standard tableau therein (i.e., the dimension of the associated irreducible representation of the symmetric group $S_{N}, N=$ $|\lambda|)$. The doubly specialized tau function

$$
\tau(0, \boldsymbol{t},-1,0,0, \ldots)=\sum_{\lambda \in \mathcal{P}} \frac{\operatorname{dim} \lambda}{|\lambda| !} Q^{|\lambda|} e^{\beta \kappa(\lambda) / 2} S_{\lambda}(\boldsymbol{t})
$$

reproduces a generating function of the single Hurwitz numbers. Note that this is a tau function of the KP hierarchy with the fermionic expression

$$
\tau(0, \boldsymbol{t},-1,0,0, \ldots)=\left\langle 0\left|\gamma_{+}(\boldsymbol{t}) Q^{L_{0}} e^{\beta K / 2} e^{J_{-1}}\right| 0\right\rangle .
$$

\section{Melting crystal models}

\subsection{Statistical model of random 3D Young diagrams}

The simplest melting crystal model [65] has a single parameter $q$ in the range $0<q<1$ (or just a formal variable). The partition function is the sum

$$
Z=\sum_{\pi \in \mathcal{P} \mathcal{P}} q^{|\pi|}
$$

of the Boltzmann weight $q^{|\pi|}$ over the set $\mathcal{P} \mathcal{P}$ of all plane partitions. The plane partition

$$
\pi=\left(\pi_{i j}\right)_{i, j=1}^{\infty}=\left(\begin{array}{ccc}
\pi_{11} & \pi_{12} & \cdots \\
\pi_{21} & \pi_{22} & \cdots \\
\vdots & \vdots & \ddots
\end{array}\right), \quad \pi_{i+1, j} \leq \pi_{i j} \geq \pi_{i, j+1},
$$


represent a 3D Young diagram in the first octant of the $x y z$-space. $\pi_{i j}$ is the height of the stacks of unit cubes on the unit square $[i-1, i] \times[j-1, j]$ of the $x y$-plane. $|\pi|$ denotes the volume of the 3D Young diagram, i.e.,

$$
|\pi|=\sum_{i, j=1}^{\infty} \pi_{i j}
$$

By the method of diagonal slicing [65], the sum (4.1) over the set of plane partitions can be converted to the sum

$$
Z=\sum_{\lambda \in \mathcal{P}} s_{\lambda}\left(q^{-\rho}\right)^{2}
$$

over the set of ordinary partitions. The building block $s_{\lambda}\left(q^{-\rho}\right)$ of the Boltzmann weight is the special value of the infinite-variate Schur function $s_{\lambda}(\boldsymbol{x})$ at

$$
\boldsymbol{x}=q^{-\rho}=\left(q^{1 / 2}, q^{3 / 2}, \ldots, q^{i-1 / 2}, \ldots\right) .
$$

This is a kind of "principal specialization" of $s_{\lambda}(\boldsymbol{x})$ [71], and can be computed by the hook-length formula

$$
s_{\lambda}\left(q^{-\rho}\right)=\frac{q^{-\kappa(\lambda) / 4}}{\prod_{(i, j) \in \lambda}\left(q^{-h(i, j) / 2}-q^{h(i, j) / 2}\right)} .
$$

Note that this formula is a $q$-analogue of (3.25) for $S_{\lambda}(1,0,0, \ldots)$.

Let us introduce another parameter $Q$, a discrete variable $s \in \mathbb{Z}$ and an infinite number of continuous variables $\boldsymbol{t}=\left(t_{1}, t_{2}, \ldots\right)$, and deform (4.2) as

$$
Z(s, \boldsymbol{t})=\sum_{\lambda \in \mathcal{P}} s_{\lambda}\left(q^{-\rho}\right)^{2} Q^{|\lambda|+s(s+1) / 2} e^{\phi(\lambda, s, \boldsymbol{t})} .
$$

$Q^{|\lambda|+s(s+1) / 2}$ is the same factor as inserted in the tau function (3.23) of the double Hurwitz numbers. $\phi(\lambda, s, \boldsymbol{t})$ is a linear combination

$$
\phi(\lambda, s, \boldsymbol{t})=\sum_{k=1}^{\infty} t_{k} \phi_{k}(\lambda, s)
$$

of the external potentials

$$
\phi_{k}(\lambda, s)=\sum_{i=1}^{\infty}\left(q^{k\left(\lambda_{i}-i+1+s\right)}-q^{k(-i+1+s)}\right)+\frac{1-q^{k s}}{1-q^{k}} q^{s},
$$

and $t_{k}$ 's play the role of coupling constants of these potentials. Note that the sum on the right hand side of (4.5) is a finite sum, because only a finite number of $\lambda_{i}$ 's are non-zero. (4.4) is related to $5 \mathrm{D} \mathcal{N}=1$ supersymmetric $U(1)$ 
Yang-Mills theory [66]. The external potentials represent the contribution of Wilson loops along the fifth dimension therein [60].

Let us mention that these external potentials are obtained from the apparently divergent (as far as $|q|<1$ ) expression

$$
\phi_{k}(\lambda, s)=\sum_{i=1}^{\infty} q^{k\left(\lambda_{i}-i+1+s\right)}-\sum_{i=1}^{\infty} q^{k(-i+1)}
$$

by recombination of terms as

$$
\phi_{k}(\lambda, s)=\sum_{i=1}^{\infty}\left(q^{k\left(\lambda_{i}-i+1+s\right)}-q^{k(-i+1+s)}\right)+\sum_{i=1}^{\infty} q^{k(-i+1+s)}-\sum_{i=1}^{\infty} q^{k(-i+1)} .
$$

The difference of the last two sums, too, thereby becomes a finite sum:

$$
\sum_{i=1}^{\infty} q^{k(-i+1+s)}-\sum_{i=1}^{\infty} q^{k(-i+1)}=\left\{\begin{array}{cc}
\sum_{i=1}^{s} q^{k(-i+1+s)} & \text { if } s>0 \\
0 & \text { if } s=0 \\
-\sum_{i=1}^{s} q^{k(-i+1)} & \text { if } s<0
\end{array}\right\}=\frac{1-q^{k s}}{1-q^{k}} q^{s}
$$

A similar prescription is used in the computation (3.20) of the factor $\langle s|g| s\rangle$ in hypergeometric tau functions. These computations are related to normal ordering of fermion bilinears.

It is this deformed partition function $Z(s, \boldsymbol{t})$ that is shown to be related to the 1D Toda hierarchy. To this end, we use a fermionic expression of $Z(s, \boldsymbol{t})$. Before showing this expression, let us present another melting crystal model.

\subsection{Modified melting crystal model}

The second model is obtained by replacing the main part of the Boltzmann weight as

$$
s_{\lambda}\left(q^{-\rho}\right)^{2} \longrightarrow s_{\lambda}\left(q^{-\rho}\right) s{ }_{\lambda}\left(q^{-\rho}\right),
$$

where ${ }^{t} \lambda$ denotes the conjugate (or transposed) partition of $\lambda$. Namely, in place of (4.2) or its $Q$-deformed version

$$
Z=\sum_{\lambda \in \mathcal{P}} s_{\lambda}\left(q^{-\rho}\right)^{2} Q^{|\lambda|}
$$

we here consider the modified partition function

$$
Z^{\prime}=\sum_{\lambda \in \mathcal{P}} s_{\lambda}\left(q^{-\rho}\right) s_{\lambda}\left(q^{-\rho}\right) Q^{|\lambda|}
$$


and its deformations by external potentials. In view of the relation

$$
s_{{ }_{\lambda}}\left(q^{-\rho}\right)=q^{\kappa(\lambda) / 2} s_{\lambda}\left(q^{-\rho}\right)
$$

that can be derived from (4.3), one can rewrite $Z^{\prime}$ as

$$
Z^{\prime}=\sum_{\lambda \in \mathcal{P}} s_{\lambda}\left(q^{-\rho}\right)^{2} q^{\kappa(\lambda) / 2} Q^{|\lambda|} .
$$

These partition functions originate in Gromov-Witten/topological string theory of special local Calabi-Yau threefolds called "local $\mathbb{C P}^{1}$ geometry" 67 , 68]. In particular, $Z^{\prime}$ is related to the "resolved conifold", for which Brini pointed out a relation to the Ablowitz-Ladik hierarchy [69].

Let us mention that one can use the homogeneity

$$
s_{\lambda}\left(Q x_{1}, Q x_{2}, \ldots\right)=Q^{|\lambda|} s_{\lambda}\left(x_{1}, x_{2}, \ldots\right)
$$

and the Cauchy identities

$$
\begin{gathered}
\sum_{\lambda \in \mathcal{P}} s_{\lambda}\left(x_{1}, x_{2}, \ldots\right) s_{\lambda}\left(y_{1}, y_{2}, \ldots\right)=\prod_{i, j \geq 1}\left(1-x_{i} y_{j}\right)^{-1}, \\
\sum_{\lambda \in \mathcal{P}} s_{\lambda}\left(x_{1}, x_{2}, \ldots\right) s_{{ }}\left(y_{1}, y_{2}, \ldots\right)=\prod_{i, j \geq 1}\left(1+x_{i} y_{j}\right)
\end{gathered}
$$

of the Schur functions to convert these partition functions to an infinite product form:

$$
\begin{aligned}
& Z=\prod_{i, j=1}^{\infty}\left(1-Q q^{i+j-1}\right)^{-1}=\prod_{n=1}^{\infty}\left(1-Q q^{n}\right)^{-n} \\
& Z^{\prime}=\prod_{i, j=1}^{\infty}\left(1+Q q^{i+j-1}\right)=\prod_{n=1}^{\infty}\left(1+Q q^{n}\right)^{-n} .
\end{aligned}
$$

These functions are referred to as the "MacMahon function" in the literature of combinatorics and mathematical physics.

We deform $Z^{\prime}$ by two sets of external potentials $\phi_{ \pm k}(\lambda, s), k=1,2, \ldots$, with coupling constants $\boldsymbol{t}=\left(t_{1}, t_{2}, \ldots\right)$ and $\overline{\boldsymbol{t}}=\left(\bar{t}_{1}, \bar{t}_{2}, \ldots\right)$ as

$$
\begin{gathered}
Z^{\prime}(s, \boldsymbol{t}, \overline{\boldsymbol{t}})=\sum_{\lambda \in \mathcal{P}} s_{\lambda}\left(q^{-\rho}\right) s_{\mathrm{t}_{\lambda}}\left(q^{-\rho}\right) Q^{|\lambda|+s(s+1) / 2} e^{\phi(\lambda, s, \boldsymbol{t}, \overline{\boldsymbol{t}})} \\
\phi(\lambda, s, \boldsymbol{t}, \overline{\boldsymbol{t}})=\sum_{k=1}^{\infty} t_{k} \phi_{k}(\lambda, s)+\sum_{k=1}^{\infty} \bar{t}_{k} \phi_{-k}(\lambda, s) .
\end{gathered}
$$

$\phi_{-k}(\lambda, s)$ 's are defined by the same formula as (4.5) with $k$ replaced by $-k$. As it turns out, $\boldsymbol{t}$ and $\overline{\boldsymbol{t}}$ correspond to the two sets of time variables of the 2D Toda hierarchy. 


\subsection{Fermionic expression of partition functions}

To translate $Z(s, \boldsymbol{t})$ and $Z^{\prime}(s, \boldsymbol{t}, \overline{\boldsymbol{t}})$ to the language of the complex free fermion system, we need some more operators on the Fock space.

Let us introduce the new fermion bilinears

$$
H_{k}=\widehat{q^{k \Delta}}=\sum_{n \in \mathbb{Z}} q^{k n}: \psi_{-n} \psi_{n}^{*}:, \quad k \in \mathbb{Z}
$$

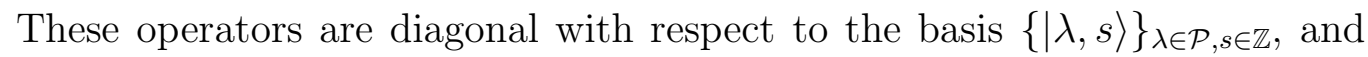
the matrix elements are nothing but the external potentials $\phi_{k}(\lambda, s)$ :

$$
\left\langle\lambda, s\left|H_{k}\right| \lambda, s\right\rangle=\phi_{k}(\lambda, s)
$$

This explains the origin of the formal expression (4.6) and its interpretation (4.5). The exponential factors in (4.4) and (4.11) can be thereby expressed as

$$
e^{\phi(\lambda, s, \boldsymbol{t})}=\left\langle\lambda, s\left|e^{H(\boldsymbol{t})}\right| \lambda, s\right\rangle, \quad e^{\phi(\lambda, s, \boldsymbol{t}, \overline{\boldsymbol{t}})}=\left\langle\lambda, s\left|e^{H(\boldsymbol{t}, \overline{\boldsymbol{t}})}\right| \lambda, s\right\rangle,
$$

where

$$
H(\boldsymbol{t})=\sum_{k=1}^{\infty} t_{k} H_{k}, \quad H(\boldsymbol{t}, \overline{\boldsymbol{t}})=\sum_{k=1}^{\infty} t_{k} H_{k}+\sum_{k=1}^{\infty} \bar{t}_{k} H_{-k} .
$$

The other building blocks of $Z(s, \boldsymbol{t})$ are similar to those of the tau function (3.23) of the double Hurwitz numbers:

$$
\begin{gathered}
s_{\lambda}\left(q^{-\rho}\right)=\left\langle s\left|\Gamma_{+}\left(q^{-\rho}\right)\right| \lambda, s\right\rangle=\left\langle\lambda, s\left|\Gamma_{-}\left(q^{-\rho}\right)\right| s\right\rangle, \\
Q^{|\lambda|+s(s+1) / 2}=\left\langle\lambda, s\left|Q^{L_{0}}\right| \lambda, s\right\rangle .
\end{gathered}
$$

These building bocks are glued together by the partition of unity (3.15) to construct the following fermionic formula of $Z(s, \boldsymbol{t})$ :

$$
Z(s, \boldsymbol{t})=\left\langle s\left|\Gamma_{+}\left(q^{-\rho}\right) Q^{L_{0}} e^{H(\boldsymbol{t})} \Gamma_{-}\left(q^{-\rho}\right)\right| s\right\rangle
$$

To derive a similar fermionic formula of $Z^{\prime}(s, \boldsymbol{t}, \overline{\boldsymbol{t}})$, we use the following variants of $\Gamma_{ \pm}(\boldsymbol{x})$ [89]:

$$
\begin{gathered}
\Gamma_{ \pm}^{\prime}(\boldsymbol{x})=\prod_{i \geq 1} \Gamma_{ \pm}^{\prime}\left(x_{i}\right), \quad \boldsymbol{x}=\left(x_{1}, x_{2}, \ldots\right) \\
\Gamma_{ \pm}^{\prime}(z)=\exp \left(-\sum_{k=1}^{\infty} \frac{(-z)^{k}}{k} J_{ \pm k}\right)
\end{gathered}
$$

The matrix elements of these modified vertex operators, too, are related to the skew Schur functions except that they are labelled by conjugate partitions:

$$
\left\langle\lambda, s\left|\Gamma_{-}^{\prime}(\boldsymbol{x})\right| \mu, s\right\rangle=\left\langle\mu, s\left|\Gamma_{+}^{\prime}(\boldsymbol{x})\right| \lambda, s\right\rangle=s{ }^{\mathrm{t}} \lambda /{ }^{\mathrm{t}} \mu(\boldsymbol{x})
$$


Thus the following fermionic formula of $Z^{\prime}(s, \boldsymbol{t}, \overline{\boldsymbol{t}})$ can be obtained in the same way as the case of $Z(s, \boldsymbol{t})$ :

$$
Z^{\prime}(s, \boldsymbol{t}, \overline{\boldsymbol{t}})=\left\langle s\left|\Gamma_{+}\left(q^{-\rho}\right) Q^{L_{0}} e^{H(\boldsymbol{t}, \overline{\boldsymbol{t}})} \Gamma_{-}^{\prime}\left(q^{-\rho}\right)\right| s\right\rangle .
$$

These fermionic formulae resemble the fermionic expression of the stationary Gromov-Witten invariants of $\mathbb{C P}^{1}$ [47, 48] and the instanton partition functions of $4 \mathrm{D} \mathcal{N}=2$ supersymmetric gauge theories [53, 54, 55, 56]. We use these formulae to show that $Z(s, \boldsymbol{t})$ and $Z^{\prime}(s, \boldsymbol{t}, \overline{\boldsymbol{t}})$ are related to tau functions of the 2D Toda hierarchy.

\section{Integrable structures of melting crystal mod- els}

\subsection{Quantum torus algebra and shift symmetries}

Although the fermionic formulae (4.14), (4.16) of the partition functions of the melting crystal mode resemble the fermionic formula (3.11) of Toda tau functions, they have manifestly different structures. In particular, it is $H_{k}$ 's rather than $J_{k}$ 's that generate deformations of the partition functions. We use special algebraic relations connecting $H_{k}$ 's and $J_{k}$ 's to convert the partition functions to Toda tau functions. These algebraic relations, referred to as "shift symmetries", are formulated in the language of a subalgebra in $\widehat{\mathrm{gl}}(\infty)$.

This subalgebra is spanned by the fermion bilinears

$$
V_{m}^{(k)}=q^{-k m / 2} \widehat{\Lambda^{m} q^{k \Delta}}=q^{-k m / 2} \sum_{n \in \mathbb{Z}} q^{k n}: \psi_{m-n} \psi_{n}^{*}:, \quad k, m \in \mathbb{Z} .
$$

This is substantially the same fermionic realization of the quantum torus algebra that are used in the work of Okounkov and Pandharipande on $\mathbb{C P}^{1}$ Gromov-Witten theory [47, 48]. $V_{m}^{(k)}$ 's satisfy the commutation relations

$$
\left[V_{m}^{(k)}, V_{n}^{(l)}\right]=\left(q^{(l m-k n) / 2}-q^{(k n-l m) / 2}\right)\left(V_{m+n}^{(k+l)}-\frac{q^{k+l}}{1-q^{k+l}} \delta_{m+n, 0}\right)
$$

for $k$ and $l$ with $k+l \neq 0$ and

$$
\left[V_{m}^{(k)}, V_{n}^{(-k)}\right]=\left(q^{-k(m+n)}-q^{k(m+n)}\right) V_{m+n}^{(0)}+m \delta_{m+n, 0}
$$

$H_{k}$ 's and $J_{k}$ 's are particular elements among $V_{m}^{(k)}$ 's:

$$
H_{k}=V_{0}^{(k)}, \quad J_{k}=V_{k}^{(0)} .
$$

We have the following three types of shift symmetries [60, 61, 63]: 
(i) For $k>0$ and $m \in \mathbb{Z}$,

$$
\begin{aligned}
\Gamma_{-}\left(q^{-\rho}\right) \Gamma_{+}\left(q^{-\rho}\right) & \left(V_{m}^{(k)}-\frac{q^{k}}{1-q^{k}} \delta_{m, 0}\right) \\
= & (-1)^{k}\left(V_{m+k}^{(k)}-\frac{q^{k}}{1-q^{k}} \delta_{m+k, 0}\right) \Gamma_{-}\left(q^{-\rho}\right) \Gamma_{+}\left(q^{-\rho}\right) .
\end{aligned}
$$

(ii) For $k>0$ and $m \in \mathbb{Z}$,

$$
\begin{aligned}
\Gamma_{-}^{\prime}\left(q^{-\rho}\right) \Gamma_{+}^{\prime}\left(q^{-\rho}\right) & \left(V_{m}^{(-k)}+\frac{1}{1-q^{k}} \delta_{m, 0}\right) \\
& =\left(V_{m+k}^{(-k)}+\frac{1}{1-q^{k}} \delta_{m+k, 0}\right) \Gamma_{-}^{\prime}\left(q^{-\rho}\right) \Gamma_{+}^{\prime}\left(q^{-\rho}\right)
\end{aligned}
$$

(iii) For $k, m \in \mathbb{Z}$,

$$
V_{m}^{(k)} q^{K / 2}=q^{-m / 2} q^{K / 2} V_{m}^{(k+m)} .
$$

Note that the indices of $V_{m}^{(k)}$ 's are literally shifted after exchanging the order of operator product with $\Gamma_{-}\left(q^{-\rho}\right) \Gamma_{+}\left(q^{-\rho}\right), \Gamma_{-}^{\prime}\left(q^{-\rho}\right) \Gamma_{+}^{\prime}\left(q^{-\rho}\right)$ and $q^{K / 2}$.

In the earlier work [60, 61, 63, we used the slightly different fermion bilinear

$$
W_{0}=\sum_{n \in \mathbb{Z}} n: \psi_{-n} \psi_{n}^{*}:
$$

and the algebraic relation

$$
V_{m}^{(k)} q^{W_{0} / 2}=q^{W_{0} / 2} V_{m}^{(k+m)}
$$

in place of $K$ and (5.5). This difference does not affect the essential part of the whole story.

\section{$5.2 Z(s, t)$ as tau function}

Let us explain how to convert the partition function $Z(s, \boldsymbol{t})$ of the first melting crystal model to a tau function of the 1D Toda hierarchy with the aid of the foregoing shift symmetries [60, 61].

The first step is to insert apparently redundant operators among $\langle s|| s$, and the operator product in between:

$$
\begin{aligned}
Z(s, \boldsymbol{t})=q^{-\left(4 s^{3}-s\right) / 12}\langle s| q^{K / 2} \Gamma_{-}\left(q^{-\rho}\right) \Gamma_{+} & \left(q^{-\rho}\right) e^{H(\boldsymbol{t})} \\
& \times Q^{L_{0}} \Gamma_{-}\left(q^{-\rho}\right) \Gamma_{+}\left(q^{-\rho}\right) q^{K / 2}|s\rangle .
\end{aligned}
$$


This is based on the identities

$$
\begin{array}{ll}
\langle s| q^{K / 2}=q^{\left(4 s^{3}-s\right) / 24}\langle s|, & \langle s| \Gamma_{-}\left(q^{-\rho}\right)=\langle s|, \\
q^{K / 2}|s\rangle=q^{\left(4 s^{3}-s\right) / 24}|s\rangle, & \Gamma_{+}\left(q^{-\rho}\right)|s\rangle=|s\rangle
\end{array}
$$

that can be derived from (3.22) and the fact that $\langle s| J_{-k}=0$ and $J_{k}|s\rangle=0$ for $k>0$. Also note that the order of $Q^{L_{0}}$ and $e^{H(\boldsymbol{t})}$, which are commutative, is reversed.

The second step is to apply the shift symmetries. The first set (5.3) of shift symmetries, specialized to $m=0$ and $k>0$, yields the identity

$$
\Gamma_{-}\left(q^{-\rho}\right) \Gamma_{+}\left(q^{-\rho}\right)\left(H_{k}-\frac{q^{k}}{1-q^{k}}\right)=(-1)^{k} V_{k}^{(k)} \Gamma_{-}\left(q^{-\rho}\right) \Gamma_{+}\left(q^{-\rho}\right)
$$

that connects $V_{0}^{(k)}=H_{k}$ and $V_{k}^{(k)}$. The third set (5.5) of shift symmetries imply the relation

$$
V_{k}^{(k)}=q^{k / 2} q^{-K / 2} J_{k} q^{K / 2}
$$

between $V_{k}^{(k)}$ and $V_{k}^{(0)}=J_{k}$. Thus $H_{k}-q^{k} /\left(1-q^{k}\right)$ and $J_{k}$ turn out to satisfy the intertwining relation

$$
q^{K / 2} \Gamma_{-}\left(q^{-\rho}\right) \Gamma_{+}\left(q^{-\rho}\right)\left(H_{k}-\frac{q^{k}}{1-q^{k}}\right)=\left(-q^{1 / 2}\right)^{k} J_{k} q^{K / 2} \Gamma_{-}\left(q^{-\rho}\right) \Gamma_{+}\left(q^{-\rho}\right)
$$

This relation can be exponentiated as

$$
\begin{aligned}
q^{K / 2} \Gamma_{-}\left(q^{-\rho}\right) \Gamma_{+}\left(q^{-\rho}\right) \exp & \left(\sum_{k=1}^{\infty} t_{k}\left(H_{k}-\frac{q^{k}}{1-q^{k}}\right)\right) \\
& =\exp \left(\sum_{k=1}^{\infty}\left(-q^{1 / 2}\right)^{k} t_{k} J_{k}\right) q^{K / 2} \Gamma_{-}\left(q^{-\rho}\right) \Gamma_{+}\left(q^{-\rho}\right) .
\end{aligned}
$$

We can thus rewrite the first half of the operator product in (5.6) as

$$
\begin{aligned}
& q^{K / 2} \Gamma_{-}\left(q^{-\rho}\right) \Gamma_{+}\left(q^{-\rho}\right) e^{H(\boldsymbol{t})}=\exp \left(\sum_{k=1}^{\infty} \frac{q^{k} t_{k}}{1-q^{k}}\right) \\
& \times \exp \left(\sum_{k=1}^{\infty}\left(-q^{1 / 2}\right)^{k} t_{k} J_{k}\right) q^{K / 2} \Gamma_{-}\left(q^{-\rho}\right) \Gamma_{+}\left(q^{-\rho}\right) .
\end{aligned}
$$


Plugging (5.7) into (5.6), we obtain the following expression of $Z(s, \boldsymbol{t})$ :

$$
\begin{aligned}
Z(s, \boldsymbol{t})=q^{-\left(4 s^{3}-s\right) / 12} \exp \left(\sum_{k=1}^{\infty} \frac{q^{k} t_{k}}{1-q^{k}}\right) & \\
& \times\left\langle s\left|\exp \left(\sum_{k=1}^{\infty}\left(-q^{1 / 2}\right)^{k} t_{k} J_{k}\right) g\right| s\right\rangle,
\end{aligned}
$$

where

$$
g=q^{K / 2} \Gamma_{-}\left(q^{-\rho}\right) \Gamma_{+}\left(q^{-\rho}\right) Q^{L_{0}} \Gamma_{-}\left(q^{-\rho}\right) \Gamma_{+}\left(q^{-\rho}\right) q^{K / 2} .
$$

Let us note that this expression is slightly different from the one presented in the previous papers [60, 61, because we use $K$ in place of $W_{0}$ in (5.6).

In much the same way, moving $e^{H(\boldsymbol{t})}$ to the right of $\Gamma_{-}\left(q^{-\rho}\right) \Gamma_{+}\left(q^{-\rho}\right) q^{K / 2}$ in (5.6), we can derive another expression of $Z(s, \boldsymbol{t})$ :

$$
\begin{aligned}
Z(s, \boldsymbol{t})=q^{-\left(4 s^{3}-s\right) / 12} \exp \left(\sum_{k=1}^{\infty} \frac{q^{k} t_{k}}{1-q^{k}}\right) & \\
& \times\left\langle s\left|g \exp \left(\sum_{k=1}^{\infty}\left(-q^{1 / 2}\right)^{k} t_{k} J_{-k}\right)\right| s\right\rangle .
\end{aligned}
$$

Actually, as one can show with the aid of the shift symmetries, the operator (5.9) connects $J_{k}$ 's and $J_{-k}$ 's as

$$
J_{k} g=g J_{-k}, \quad k=1,2, \ldots
$$

This explains why $Z(s, \boldsymbol{t})$ has the two apparently different expressions (5.8) and (5.10).

Apart from the prefactors and the rescaling $t_{k} \rightarrow\left(-q^{1 / 2}\right)^{k} t_{k}$ of the time variables, the essential part of the right side of (5.8) and (5.10) is the function

$$
\tau(s, \boldsymbol{t})=\left\langle s\left|\gamma_{+}(\boldsymbol{t}) g\right| s\right\rangle=\left\langle s\left|g \gamma_{-}(\boldsymbol{t})\right| s\right\rangle .
$$

By the symmetry (5.11) of $g$, the associated 2D Toda tau function reduces to this function:

$$
\tau(s, \boldsymbol{t}, \overline{\boldsymbol{t}})=\left\langle s\left|\gamma_{+}(\boldsymbol{t}) g \gamma_{-}(-\overline{\boldsymbol{t}})\right| s\right\rangle=\tau(s, \boldsymbol{t}-\overline{\boldsymbol{t}}) .
$$

This means that $\tau(s, \boldsymbol{t})$ is a tau function of the 1D Toda hierarchy.

Remark 1. The exponential functions in (5.8) and (5.10) can be absorbed by redefinition of the tau function replacing

$$
g \rightarrow \tilde{g}=\exp \left(\sum_{k=1}^{\infty} \frac{\left(-q^{1 / 2}\right)^{k}}{k\left(1-q^{k}\right)} J_{-k}\right) g \exp \left(\sum_{k=1}^{\infty} \frac{\left(-q^{1 / 2}\right)^{k}}{k\left(1-q^{k}\right)} J_{k}\right)
$$


This is a consequence of the identities

$$
\begin{aligned}
& \exp \left(\sum_{k=1}^{\infty} \frac{q^{k} t_{k}}{1-q^{k}}\right) \exp \left(\sum_{k=1}^{\infty}\left(-q^{1 / 2}\right)^{k} t_{k} J_{k}\right) \exp \left(\sum_{k=1}^{\infty}\left(-q^{1 / 2}\right)^{k} t_{k} J_{k}\right) \exp \left(\sum_{k=1}^{\infty} \frac{\left(-q^{1 / 2}\right)^{k}}{k\left(1-q^{k}\right)} J_{-k}\right) \\
& \exp \left(\sum_{k=1}^{\infty} \frac{q^{k} t_{k}}{1-q^{k}}\right) \exp \left(\sum_{k=1}^{\infty}\left(-q^{1 / 2}\right)^{k} t_{k} J_{-k}\right) \\
&=\exp \left(\sum_{k=1}^{\infty} \frac{\left(-q^{1 / 2}\right)^{k}}{k\left(1-q^{k}\right)} J_{k}\right) \exp \left(\sum_{k=1}^{\infty}\left(-q^{1 / 2}\right)^{k} t_{k} J_{-k}\right)
\end{aligned}
$$

that can be deduced from the commutation relations (3.5) of $J_{ \pm k}$ 's. Note that the new generating operator $\tilde{g}$, too, satisfies the $1 \mathrm{D}$ reduction condition

$$
J_{k} \tilde{g}=\tilde{g} J_{-k}, \quad k=1,2, \ldots
$$

It is also remarkable that the two operators in the transformation $g \rightarrow \tilde{g}$ are related to the vertex operators:

$$
\exp \left(\sum_{k=1}^{\infty} \frac{\left(-q^{1 / 2}\right)^{k}}{k\left(1-q^{k}\right)} J_{ \pm k}\right)=\Gamma_{ \pm}^{\prime}\left(q^{-\rho}\right)^{-1}
$$

Remark 2. There is an another way to avoid the exponential factors in (5.8) and (5.10). These factors disappear if the external potentials $\phi_{k}(\lambda)$ are modified as

$$
\phi_{k}(\lambda, s)=\sum_{i=1}^{\infty}\left(q^{k\left(\lambda_{i}-i+1+s\right)}-q^{k(-i+1+s)}\right)-\frac{q^{k s}}{1-q^{k}} q^{s},
$$

namely, if the constant term $q^{k} /\left(1-q^{k}\right)$ is subtracted from $\phi_{k}(\lambda)$. This amounts to modifying the definition (4.12) of $H_{k}$ as

$$
H_{k}=\widehat{q^{k \Delta}}-\frac{q^{k}}{1-q^{k}}
$$

The foregoing computations with the aid of the shift symmetries, too, can be slightly simplified by this redefinition of $H_{k}$ 's. Note that the prefactor $q^{-\left(4 s^{3}-s\right) / 12}$ cannot be removed by this modification. 


\section{3 $Z^{\prime}(s, t, \bar{t})$ as tau function}

The partition function $Z^{\prime}(s, \boldsymbol{t}, \overline{\boldsymbol{t}})$ of the second melting crystal model can be treated in a parallel manner. Let us show an outline of the computations 63 .

The first step is to rewrite the fermionic expression (4.16) as follows:

$$
\begin{aligned}
Z^{\prime}(s, \boldsymbol{t}, \overline{\boldsymbol{t}})=\langle s| q^{K / 2} \Gamma_{-}\left(q^{-\rho}\right) \Gamma_{+} & \left(q^{-\rho}\right) e^{H(\boldsymbol{t})} \\
& \times Q^{L_{0}} e^{\bar{H}(\overline{\boldsymbol{t}})} \Gamma_{-}^{\prime}\left(q^{-\rho}\right) \Gamma_{+}^{\prime}\left(q^{-\rho}\right) q^{-K / 2}|s\rangle,
\end{aligned}
$$

where

$$
\bar{H}(\overline{\boldsymbol{t}})=\sum_{k=1}^{\infty} \bar{t}_{k} J_{-k} .
$$

Note that we have split $e^{H(\boldsymbol{t}, \overline{\boldsymbol{t}})}$ into $e^{H(\boldsymbol{t})}$ and $e^{\bar{H}(\overline{\boldsymbol{t}})}$, and inserted $\Gamma_{+}^{\prime}\left(q^{-\rho}\right) q^{-K / 2}$ in place of $\Gamma_{+}\left(q^{-\rho}\right) q^{K / 2}$ to the right end of the operator product.

The second step is to transfer $e^{H(\boldsymbol{t})}$ and $e^{\bar{H}(\overline{\boldsymbol{t}})}$ to the left and right ends, respectively, with the aid of the shift symmetries. Computations for $e^{H(\boldsymbol{t})}$ are exactly the same as the case of $Z(s, \boldsymbol{t})$. To transfer $e^{\bar{H}(\overline{\boldsymbol{t}})}$, we combine the shift symmetries of the second type (5.4) and the third type (5.5). This yields the relation

$$
\left(H_{-k}+\frac{1}{1-q^{k}}\right) \Gamma_{-}^{\prime}\left(q^{-\rho}\right) \Gamma_{+}^{\prime}\left(q^{-\rho}\right) q^{-K / 2}=\Gamma_{-}^{\prime}\left(q^{-\rho}\right) \Gamma_{+}^{\prime}\left(q^{-\rho}\right) q^{-K / 2} J_{-k}
$$

connecting $H_{-k}+1 /\left(1-q^{k}\right)$ and $J_{-k}$. Exponentiating this relation, we obtain the following counterpart of (5.17):

$$
\begin{aligned}
e^{\bar{H}(\overline{\boldsymbol{t}})} \Gamma_{-}^{\prime}\left(q^{-\rho}\right) \Gamma_{+}^{\prime}\left(q^{-\rho}\right) q^{-K / 2}=\exp \left(-\sum_{k=1}^{\infty} \frac{\bar{t}_{k}}{1-q^{k}}\right) \\
\times \Gamma_{-}^{\prime}\left(q^{-\rho}\right) \Gamma_{+}^{\prime}\left(q^{-\rho}\right) q^{-K / 2} \exp \left(\sum_{k=1}^{\infty} q^{-k / 2} \bar{t}_{k} J_{-k}\right) .
\end{aligned}
$$

Plugging (5.7) and (5.17) into (5.16), we can rewrite $Z^{\prime}(s, \boldsymbol{t}, \overline{\boldsymbol{t}})$ as

$$
\begin{aligned}
Z^{\prime}(s, \boldsymbol{t}, \overline{\boldsymbol{t}}) & =\exp \left(\sum_{k=1}^{\infty} \frac{q^{k} t_{k}-\bar{t}_{k}}{1-q^{k}}\right) \\
& \times\left\langle s\left|\exp \left(\sum_{k=1}^{\infty}\left(-q^{1 / 2}\right)^{k} t_{k} J_{k}\right) g \exp \left(\sum_{k=1}^{\infty} q^{-k / 2} \bar{t}_{k} J_{-k}\right)\right| s\right\rangle
\end{aligned}
$$


where

$$
g=q^{K / 2} \Gamma_{-}\left(q^{-\rho}\right) \Gamma_{+}\left(q^{-\rho}\right) Q^{L_{0}} \Gamma_{-}^{\prime}\left(q^{-\rho}\right) \Gamma_{+}^{\prime}\left(q^{-\rho}\right) q^{-K / 2} .
$$

Thus, apart from the exponential prefactor and the rescaling $t_{k} \rightarrow\left(-q^{1 / 2}\right)^{k} t_{k}$, $\bar{t}_{k} \rightarrow q^{-k / 2} \bar{t}_{k}$ of the time variables, $Z^{\prime}(s, \boldsymbol{t}, \overline{\boldsymbol{t}})$ is a tau function of the $2 \mathrm{D}$ Toda hierarchy generated by the operator (5.19).

One can find no symmetry like (5.11) for the generating operator (5.19). The associated tau function is a genuine 2D Toda tau function. Actually, this special solution of the 2D Toda hierarchy falls into the Ablowitz-Ladik hierarchy 63].

Remark 3. The exponential prefactor in (5.18) can be absorbed by replacing

$$
g \rightarrow \tilde{g}=\exp \left(\sum_{k=1}^{\infty} \frac{\left(-q^{1 / 2}\right)^{k}}{k\left(1-q^{k}\right)} J_{-k}\right) g \exp \left(-\sum_{k=1}^{\infty} \frac{q^{k / 2}}{k\left(1-q^{k}\right)} J_{k}\right) .
$$

Alternatively, one can remove this prefactor by subtracting the constant terms $q^{ \pm k} /\left(1-q^{ \pm k}\right)$ from the external potentials $\phi_{ \pm k}(\lambda)$ as shown in (5.14). The operators $H_{ \pm k}$ are accordingly modified as shown in (5.15).

\subsection{Shift symmetries in matrix formalism}

We here turn to a digression on the quantum torus algebra and the shift symmetries. This is not just a digression, but closely related to the subsequent consideration in the perspective of the Lax formalism.

The foregoing quantum torus Lie algebra and shift symmetries can be translated to the language of infinite matrices by the correspondence $A \leftrightarrow \widehat{A}$ between $\mathbb{Z} \times \mathbb{Z}$ matrices and fermion bilinears. This matrix formalism enables us to use the associative product of matrices as well. In particular, the matrix representation $\boldsymbol{V}_{m}^{(k)}$ of $V_{m}^{(k)}$ are expressed in term of $\Lambda$ and $\Delta$ as

$$
\boldsymbol{V}_{m}^{(k)}=q^{-k m / 2} \Lambda^{m} q^{k \Delta}
$$

Moreover, the commutation relations

$$
\left[\boldsymbol{V}_{m}^{(k)}, \boldsymbol{V}_{n}^{(l)}\right]=\left(q^{(l m-k n) / 2}-q^{(k n-l m) / 2}\right) \boldsymbol{V}_{m+n}^{(k+l)}
$$

of the centerless quantum torus Lie algebra can be derived from the so called quantum torus relation

$$
\Lambda q^{\Delta}=q q^{\Delta} \Lambda
$$

satisfied by $\Lambda$ and $q^{\Delta}$, which generate an associative quantum torus algebra.

Moreover, the vertex operators $\Gamma_{ \pm}\left(q^{-\rho}\right)$ and $\Gamma_{ \pm}^{\prime}\left(q^{-\rho}\right)$ reveals a hidden link with the notion of quantum dilogarithmic functions [90, 91] through the matrix representation. Such a Clifford operator, too, have the associated matrix 
representation through the exponentiation $e^{A} \leftrightarrow e^{\hat{A}}$ of the Lie algebraic correspondence $A \leftrightarrow \hat{A}$. The fundamental vertex operators $\Gamma_{ \pm}(x)$ and $\Gamma_{ \pm}^{\prime}(x)$ thereby correspond to the following matrices:

$$
\begin{gathered}
\boldsymbol{\Gamma}_{ \pm}(x)=\exp \left(\sum_{k=1}^{\infty} \frac{x^{k}}{k} \Lambda^{ \pm k}\right)=\left(1-x \Lambda^{ \pm 1}\right)^{-1}, \\
\Gamma_{ \pm}^{\prime}(x)=\exp \left(-\sum_{k=1}^{\infty} \frac{(-x)^{k}}{k} \Lambda^{ \pm}\right)=\left(1+x \Lambda^{ \pm 1}\right) .
\end{gathered}
$$

Consequently, the matrix representation of $\Gamma_{ \pm}\left(q^{-\rho}\right)$ and $\Gamma_{ \pm}^{\prime}\left(q^{-\rho}\right)$ become an infinite product of these matrices specialized to $x=q^{i-1 / 2}$ :

$$
\boldsymbol{\Gamma}_{ \pm}\left(q^{-\rho}\right)=\prod_{i=1}^{\infty}\left(1-q^{i-1 / 2} \Lambda^{ \pm 1}\right)^{-1}, \quad \boldsymbol{\Gamma}_{ \pm}^{\prime}\left(q^{-\rho}\right)=\prod_{i=1}^{\infty}\left(1+q^{i-1 / 2} \Lambda^{ \pm 1}\right) .
$$

These infinite products may be thought of as matrix-valued quantum dilogarithmic functions in the sense of Faddeev et al.

We thus find the following matrix analogues of the shift symmetries:

(i) For $k>0$ and $m \in \mathbb{Z}$,

$$
\boldsymbol{\Gamma}_{-}\left(q^{-\rho}\right) \boldsymbol{\Gamma}_{+}\left(q^{-\rho}\right) \boldsymbol{V}_{m}^{(k)}=(-1)^{k} \boldsymbol{V}_{m+k}^{(k)} \boldsymbol{\Gamma}_{-}\left(q^{-\rho}\right) \boldsymbol{\Gamma}_{+}\left(q^{-\rho}\right) .
$$

(ii) For $k>0$ and $m \in \mathbb{Z}$,

$$
\boldsymbol{\Gamma}_{-}^{\prime}\left(q^{-\rho}\right) \boldsymbol{\Gamma}_{+}^{\prime}\left(q^{-\rho}\right) \boldsymbol{V}_{m}^{(-k)}=\boldsymbol{V}_{m+k}^{(-k)} \boldsymbol{\Gamma}_{-}^{\prime}\left(q^{-\rho}\right) \boldsymbol{\Gamma}_{+}^{\prime}\left(q^{-\rho}\right)
$$

(iii) For $k, m \in \mathbb{Z}$,

$$
\boldsymbol{V}_{m}^{(k)} q^{(\Delta-1 / 2)^{2} / 2}=q^{-m / 2} q^{(\Delta-1 / 2)^{2} / 2} \boldsymbol{V}_{m}^{(k+m)} .
$$

These matrix analogues of the shift symmetries can be derived from the matrix representation (5.20), (5.24) of $V_{m}^{(k)}$ 's and the vertex operators by straightforward computations using the quantum torus relation (5.22) [63].

\subsection{Perspectives in Lax formalism}

Let us return to the melting crystal models, and consider the associated special solutions of the 2D Toda hierarchy in the Lax formalism. The goal is to show that the Lax operators $L, \bar{L}$ satisfy the reduction conditions (2.21) and (2.26) to the 1D Toda and Ablowitz-Ladik hierarchies [63]. The reasoning can be outlined as follows. 
1. It is enough to show that the initial values of the Lax operators at $\boldsymbol{t}=\overline{\boldsymbol{t}}=\mathbf{0}$ satisfy the reduction condition (2.21) and (2.26), because these factorized forms are preserved by the time evolutions of the $2 \mathrm{D}$ Toda hierarchy.

2. One can explicitly solve the factorization problem (2.29) for these cases at the initial time. The initial values of the dressing operators are written in terms of the matrix representation (5.24) of the vertex operators and some other simple matrices.

3. The initial values of the Lax operators can be computed with the aid of these matrices, and turn out to take the forms as shown in (2.21) and (2.26).

\subsubsection{First melting crystal model}

The generating operator (5.9) in this case corresponds to a matrix of the form

$$
U=q^{(\Delta-1 / 2)^{2} / 2} \boldsymbol{\Gamma}_{-}\left(q^{-\rho}\right) \boldsymbol{\Gamma}_{+}\left(q^{-\rho}\right) Q^{\Delta} \boldsymbol{\Gamma}_{-}\left(q^{-\rho}\right) \boldsymbol{\Gamma}_{+}\left(q^{-\rho}\right) q^{(\Delta-1 / 2)^{2} / 2} .
$$

One can use the identities

$$
Q^{\Delta} \Lambda^{n} Q^{-\Delta}=Q^{-n} \Lambda^{n}, \quad Q^{-\Delta} \Lambda^{n} Q^{\Delta}=Q^{n} \Lambda^{n}
$$

to rewrite the triple product in the middle as

$$
U=q^{(\Delta-1 / 2)^{2} / 2} \boldsymbol{\Gamma}_{-}\left(q^{-\rho}\right) \boldsymbol{\Gamma}_{-}\left(Q q^{-\rho}\right) Q^{\Delta} \boldsymbol{\Gamma}_{+}\left(Q q^{-\rho}\right) \boldsymbol{\Gamma}_{+}\left(q^{-\rho}\right) q^{(\Delta-1 / 2)^{2} / 2} .
$$

This matrix is already factorized to a product of lower and upper triangular matrices as

$$
U=W_{0}^{-1} \bar{W}_{0}
$$

where

$$
\begin{gathered}
W_{0}=q^{(\Delta-1 / 2)^{2} / 2} \boldsymbol{\Gamma}_{-}\left(Q q^{-\rho}\right)^{-1} \boldsymbol{\Gamma}_{-}\left(q^{-\rho}\right)^{-1} q^{-(\Delta-1 / 2)^{2} / 2}, \\
\bar{W}_{0}=q^{(\Delta-1 / 2)^{2} / 2} Q^{\Delta} \boldsymbol{\Gamma}_{+}\left(Q q^{-\rho}\right) \boldsymbol{\Gamma}_{+}\left(q^{-\rho}\right) q^{(\Delta-1 / 2)^{2} / 2} .
\end{gathered}
$$

This means that $W_{0}$ and $\bar{W}_{0}$ are the initial values $\left.W\right|_{\boldsymbol{t}=\overline{\boldsymbol{t}}=\mathbf{0}},\left.\bar{W}\right|_{\boldsymbol{t}=\overline{\boldsymbol{t}}=\mathbf{0}}$ of the dressing operators determined by the generating matrix (5.28).

One can compute the initial values

$$
L_{0}=\left.L\right|_{\boldsymbol{t}=\overline{\boldsymbol{t}}=\mathbf{0}}=W_{0} \Lambda W_{0}^{-1}, \quad \bar{L}_{0}^{-1}=\left.\bar{L}\right|_{\boldsymbol{t}=\overline{\boldsymbol{t}}=\mathbf{0}} ^{-1}=\bar{W}_{0} \Lambda^{-1} \bar{W}_{0}^{-1}
$$

of the Lax operators from these explicit forms of $W_{0}$ and $\bar{W}_{0}$ as follows. 
The first step for computing $L_{0}$ is to uses the identity

$$
q^{-(\Delta-1 / 2)^{2} / 2} \Lambda q^{(\Delta / 1 / 2)^{2} / 2}=q^{\Delta} \Lambda
$$

that is a consequence of (5.27). By this identity and the expression (5.30) of $W_{0}$, one can rewrite $L_{0}$ as

$$
L_{0}=q^{(\Delta-1 / 2)^{2} / 2} \boldsymbol{\Gamma}_{-}\left(Q q^{-\rho}\right)^{-1} \boldsymbol{\Gamma}_{-}\left(q^{-\rho}\right)^{-1} q^{\Delta} \Lambda \boldsymbol{\Gamma}_{-}\left(q^{-\rho}\right) \boldsymbol{\Gamma}_{-}\left(Q q^{-\rho}\right) q^{-(\Delta-1 / 2)^{2} / 2} .
$$

Since $\boldsymbol{\Gamma}_{-}\left(q^{-\rho}\right)$ and $\boldsymbol{\Gamma}_{-}\left(Q q^{-\rho}\right)$ are matrices of the form

$$
\boldsymbol{\Gamma}_{-}\left(q^{-\rho}\right)=\prod_{i=1}^{\infty}\left(1-q^{i-1 / 2} \Lambda^{-1}\right)^{-1}, \quad \boldsymbol{\Gamma}_{-}\left(Q q^{-\rho}\right)=\prod_{i=1}^{\infty}\left(1-Q q^{i-1 / 2} \Lambda^{-1}\right)^{-1}
$$

the matrix $\Lambda$ in front of these two matrices can be moved to the right side as

$$
\Lambda \boldsymbol{\Gamma}_{-}\left(q^{-\rho}\right) \boldsymbol{\Gamma}_{-}\left(Q q^{-\rho}\right)=\boldsymbol{\Gamma}_{-}\left(q^{-\rho}\right) \boldsymbol{\Gamma}_{-}\left(Q q^{-\rho}\right) \Lambda .
$$

One can further use the identity

$$
q^{\Delta} \Lambda^{-1} q^{-\Delta}=q \Lambda^{-1}
$$

to transfer the remaining $q^{\Delta}$ to the right as

$$
\begin{aligned}
q^{\Delta} \boldsymbol{\Gamma}_{-}\left(q^{-\rho}\right) \boldsymbol{\Gamma}_{-}\left(Q q^{-\rho}\right) & =q^{\Delta} \prod_{i=1}^{\infty}\left(1-q^{i-1 / 2} \Lambda^{-1}\right)^{-1} \prod_{i=1}^{\infty}\left(1-Q q^{i-1 / 2} \Lambda^{-1}\right)^{-1} \\
& =\prod_{i=1}^{\infty}\left(1-q^{i+1 / 2} \Lambda^{-1}\right)^{-1} \prod_{i=1}^{\infty}\left(1-Q q^{i+1 / 2} \Lambda^{-1}\right)^{-1} \cdot q^{\Delta} \\
& =\boldsymbol{\Gamma}_{-}\left(q^{-\rho}\right) \boldsymbol{\Gamma}_{-}\left(Q q^{-\rho}\right)\left(1-Q q^{1 / 2} \Lambda^{-1}\right)\left(1-q^{1 / 2} \Lambda^{-1}\right) q^{\Delta}
\end{aligned}
$$

The outcome reads

$$
L_{0}=q^{(\Delta-1 / 2)^{2} / 2}\left(1-Q q^{1 / 2} \Lambda^{-1}\right)\left(1-q^{1 / 2} \Lambda^{-1}\right) q^{\Delta} \Lambda q^{-(\Delta-1 / 2)^{2} / 2} .
$$

Lastly, by the identities

$$
\begin{gathered}
q^{(\Delta-1 / 2)^{2} / 2} \Lambda q^{-(\Delta-1 / 2)^{2} / 2}=q^{-\Delta} \Lambda, \\
q^{(\Delta-1 / 2)^{2} / 2} \Lambda^{-1} q^{-(\Delta-1 / 2)^{2} / 2}=\Lambda^{-1} q^{\Delta}=q^{-1} q^{\Delta} \Lambda^{-1}
\end{gathered}
$$

one can rewrite the last expression of $L_{0}$ as

$$
\begin{aligned}
L_{0} & =\left(1-Q q^{-1 / 2} q^{\Delta} \Lambda^{-1}\right)\left(1-q^{-1 / 2} q^{\Delta} \Lambda^{-1}\right) \Lambda \\
& =\Lambda-(Q+1) q^{-1 / 2} q^{\Delta}+Q q^{-2} q^{2 \Delta} \Lambda^{-1} .
\end{aligned}
$$

One can compute $\bar{L}_{0}^{-1}$ in much the same way, and confirm that it coincides with the expression (5.31) of $L_{0}$. This implies that the reduction condition (2.21) to the 1D Toda hierarchy is indeed satisfied. 


\subsubsection{Second melting crystal model}

The generating operator (5.19) in this case corresponds to the matrix

$$
U=q^{(\Delta-1 / 2)^{2} / 2} \boldsymbol{\Gamma}_{-}\left(q^{-\rho}\right) \boldsymbol{\Gamma}_{+}\left(q^{-\rho}\right) Q^{\Delta} \boldsymbol{\Gamma}_{-}^{\prime}\left(q^{-\rho}\right) \boldsymbol{\Gamma}_{+}^{\prime}\left(q^{-\rho}\right) q^{-(\Delta-1 / 2)^{2} / 2} .
$$

This matrix can be factorized as

$$
U=W_{0}^{-1} \bar{W}_{0}
$$

with

$$
\begin{gathered}
W_{0}=q^{(\Delta-1 / 2)^{2} / 2} \boldsymbol{\Gamma}_{-}^{\prime}\left(Q q^{-\rho}\right)^{-1} \boldsymbol{\Gamma}_{-}\left(q^{-\rho}\right)^{-1} q^{-(\Delta-1 / 2)^{2} / 2}, \\
\bar{W}_{0}=q^{(\Delta-1 / 2)^{2} / 2} Q^{\Delta} \boldsymbol{\Gamma}_{+}\left(Q q^{-\rho}\right) \boldsymbol{\Gamma}_{+}^{\prime}\left(q^{-\rho}\right) q^{-(\Delta-1 / 2)^{2} / 2} .
\end{gathered}
$$

One can compute $L_{0}$ in much the same way as the previous case, starting from the expression

$$
L_{0}=q^{(\Delta-1 / 2)^{2} / 2} \boldsymbol{\Gamma}_{-}^{\prime}\left(Q q^{-\rho}\right)^{-1} \boldsymbol{\Gamma}_{-}\left(q^{-\rho}\right)^{-1} q^{\Delta} \Lambda \boldsymbol{\Gamma}_{-}\left(q^{-\rho}\right) \boldsymbol{\Gamma}_{-}^{\prime}\left(Q q^{-\rho}\right) q^{-(\Delta-1 / 2)^{2} / 2} .
$$

This expression contains

$$
\Gamma_{-}^{\prime}\left(Q q^{-\rho}\right)=\prod_{i=1}^{\infty}\left(1+Q q^{i-1 / 2} \Lambda^{-1}\right)
$$

in place of $\boldsymbol{\Gamma}_{-}\left(Q q^{-\rho}\right)$. Consequently, the foregoing transfer procedure of $q^{\Delta}$ is modified as

$$
\begin{aligned}
q^{\Delta} \boldsymbol{\Gamma}_{-}\left(q^{-\rho}\right) \boldsymbol{\Gamma}_{-}^{\prime}\left(Q q^{-\rho}\right) & =q^{\Delta} \prod_{i=1}^{\infty}\left(1-q^{i-1 / 2} \Lambda^{-1}\right)^{-1} \prod_{i=1}^{\infty}\left(1+Q q^{i-1 / 2} \Lambda^{-1}\right) \\
& =\prod_{i=1}^{\infty}\left(1-q^{i+1 / 2} \Lambda^{-1}\right)^{-1} \prod_{i=1}^{\infty}\left(1+Q q^{i+1 / 2} \Lambda^{-1}\right) \cdot q^{\Delta} \\
& =\boldsymbol{\Gamma}_{-}\left(q^{-\rho}\right) \boldsymbol{\Gamma}_{-}^{\prime}\left(Q q^{-\rho}\right)\left(1+Q q^{1 / 2} \Lambda^{-1}\right)^{-1}\left(1-q^{1 / 2} \Lambda^{-1}\right) q^{\Delta} .
\end{aligned}
$$

The final expression of $L_{0}$ takes the quotient form

$$
L_{0}=\left(1+Q q^{-1 / 2} q^{\Delta} \Lambda^{-1}\right)^{-1}\left(1-q^{-1 / 2} q^{\Delta} \Lambda^{-1}\right) \Lambda .
$$

One can compute $\bar{L}_{0}^{-1}$ in much the same (but slightly more complicated) way starting. (5.33) and the identity

$$
q^{-(\Delta-1 / 2)^{2} / 2} \Lambda^{-1} q^{(\Delta-1 / 2)^{2} / 2}=\Lambda^{-1} q^{-\Delta}
$$


imply that $\bar{L}_{0}^{-1}$ can be expressed as

$$
\begin{aligned}
\bar{L}_{0}^{-1}=q^{(\Delta-1 / 2)^{2} / 2} Q^{\Delta} \boldsymbol{\Gamma}_{+} & \left(Q q^{-\rho}\right) \boldsymbol{\Gamma}_{+}^{\prime}\left(q^{-\rho}\right) \\
& \times \Lambda^{-1} q^{-\Delta} \boldsymbol{\Gamma}_{+}^{\prime}\left(q^{-\rho}\right)^{-1} \boldsymbol{\Gamma}_{+}\left(Q q^{-\rho}\right)^{-1} Q^{-\Delta} q^{-(\Delta-1 / 2)^{2} / 2} .
\end{aligned}
$$

The outcome of somewhat lengthy computations reads

$$
\bar{L}_{0}^{-1}=\left(1-q^{1 / 2} q^{-\Delta} \Lambda\right)^{-1}\left(1+Q^{-1} q^{1 / 2} q^{-\Delta} \Lambda\right) Q \Lambda^{-1} .
$$

It is easy to see that (5.34) and (5.35) can be rewritten as

$$
L_{0}=\tilde{C}_{0}^{-1} \tilde{B}_{0}, \quad \bar{L}_{0}^{-1}=-\tilde{B}_{0}^{-1} \tilde{C}_{0}
$$

where

$$
\tilde{B}_{0}=\Lambda-q^{-1 / 2} q^{\Delta}, \quad \tilde{C}_{0}=1+Q q^{-1 / 2} q^{\Delta} \Lambda^{-1} .
$$

This coincides with the reduced form of (2.26) except for the negative sign in the expression of $\bar{L}_{0}^{-1}$. The negative sign is harmless, because it can be absorbed by the time reversal $\overline{\boldsymbol{t}} \rightarrow-\overline{\boldsymbol{t}}$. Actually, one can express $L_{0}$ and $\bar{L}_{0}$ in the form of (2.24) as well (again with an extra negative sign) 63. Anyway, the reduction condition to the Ablowiz-Ladik hierarchy is satisfied in this case.

\section{Conclusion}

It is remarkable that the two melting crystal models repeat the same pattern of integrable structures as the Hermitian and unitary matrix models. A major difference is the fact that the partition functions of the matrix models are $s \times s$ determinants (hence the lattice coordinate $s$ therein take values in positive integers), whereas there is no such expression of the partition functions of the melting crystal models as determinants of finite size. The discrete variable $s$ of the melting crystal models enters the Boltzmann weights as a parameter. This is a main reason why we need an entirely different method to identify the underlying integrable structures.

On the other hand, the undeformed partition functions (4.7) and (4.9) of the two melting crystal models differs in just the single factor $q^{\kappa(\lambda) / 2}$. It is somewhat surprising that this tiniest modification leads to a drastic change in the underlying integrable structure. Of course this is rather natural from a geometric point of view, because the associated Calabi-Yau threefolds are different.

The shift symmetries of the quantum torus algebra lie in the heart of our method. These algebraic relations are used to transform the "diagonal" 
Hamiltonians $H_{k}=V_{0}^{(k)}$ to the "non-diagonal" generators $J_{m}$ of time evolutions of the 2D Toda hierarchy. Let us mention two other approaches to this kind of unconventional time evolutions (see also Section 3.5 of the review of Alexandrov and Zabrodin [23]).

The first one is Orlov's approach [92] to a class of KP tau functions obtained from the hypergeometric functions (3.18) by specializing the second set $\overline{\boldsymbol{t}}$ of time variables to a particular point. The special value of $S_{\lambda}(-\overline{\boldsymbol{t}})$ at that point $\overline{\boldsymbol{t}}=-\boldsymbol{a}$ becomes a determinant of the Cauchy type. The Schur function expansion of $\tau(s, \boldsymbol{t},-\boldsymbol{a})$ can be thereby reorganized to an " $\infty$-soliton solution" of the KP hierarchy in which the parameters $\boldsymbol{T}=\left(T_{1}, T_{2}, \ldots\right)$ of the generating operator (3.17) play the role of time variables.

The second approach is developed by Bettelheim et al. 93] in their research of a complex fermion system on the real line. Time evolutions of this system are generated by diagonal Hamiltonians similar to our $H_{k}$ 's except that the coefficients $q^{k n}$ of $: \psi_{-n}^{*} \psi_{n}$ : are replaced by $n^{k}$. Bettelheim et al. considered an analogue of KP and Toda tau functions in which $J_{k}$ 's and the ground states $\langle s|$ and $|s\rangle$ are replaced by $H_{k}$ 's and what they call "boundary states", $\left\langle B_{s}\right|$ and $\left|B_{s}\right\rangle$. These boundary states are generated from the vacuum states $\langle 0|$ and $|0\rangle$ by "boundary operators" $B_{s}$. The modified "tau functions" are shown to satisfy the bilinear equations of the KP and Toda hierarchies. Unfortunately, it is difficult to compare the results of Bettelheim et al. with ours literally, because the setup of the fermion system is different. Our complex free fermions live on a circle $|z|=R$ of the $z$-plane rather than the real axis. Nevertheless, it is obvious that the boundary operators $B_{s}$ play the same role as $\Gamma_{-}\left(q^{-\rho}\right) \Gamma_{+}\left(q^{-\rho}\right)$ in our approach.

We believe that the shift symmetries will be useful beyond the scope of the melting crystal models. The results reviewed in this paper should be just a small piece of possible applications. In fact, we recently applied the shift symmetries to computations of topological string theory in a special case [94. We are currently trying to find how the algebraic relations (5.3) and (5.4) are altered outside the range $k>0$. Hopefully, the shift symmetries thus extended will become a new tool of computations for various purposes.

It is also true that the shift symmetries are a very special property of the vertex operators $\Gamma_{ \pm}\left(q^{-\rho}\right)$ and $\Gamma_{ \pm}^{\prime}\left(q^{-\rho}\right)$. Until now, we have been unable to find a similar tool for the $4 \mathrm{D}$ version [53, 54, 55, 56] of $Z(s, \boldsymbol{t})$ and $Z^{\prime}(s, \boldsymbol{t}, \overline{\boldsymbol{t}})$. The aforementioned boundary operators of Bettelheim et al. might be a clue to this problem. It seems more likely that another clue is hidden in the fermionic formalism of $\mathbb{C P}^{1}$ Gromov-Witten theory developed by Okounkov and Pandharipande [47, 48]. 


\section{Acknowledgements}

I would like to thank Takashi Takebe and Toshio Nakatsu for longstanding collaboration. I am also grateful to Mark Adler, Pierre van Moerbeke, John Harnad and Sasha Orlov for having constant interests in the Toda hierarchy. Last but not least, I am indebted to Kimio Ueno for support in the earliest stage of the studies on the Toda hierarchies. This work is partly supported by the JSPS Kakenhi Grant No. 25400111 and No. 15K04912.

\section{References}

[1] M. Toda, Vibration of a chain with a non-linear interaction, J. Phys. Soc. Japan 22 (1967), 431-436.

[2] M. Toda, Wave propagation in anharmonic lattice, J. Phys. Soc. Japan 23 (1967), 501-596.

[3] R. Hirota, Exact $N$-soliton solution of a nonlinear lumped network equation, J. Phys. Soc. Japan 35 (1973), 286-288.

[4] M. Hénon, Integrals of the Toda lattice, Phys. Rev. B9 (1974), 19211923.

[5] H. Flaschka, The Toda lattice I, Existence of integrals, Phys. Rev. B9 (1974), 1924-1925.

[6] H. Flaschka, On the Toda lattice II, Inverse scattering solution, Prog. Theor. Phys. 51 (1974), 703-716.

[7] S. V. Manakov, Complete integrability and stochastization of discrete dynamical systems, Soviet Phys. JETP 40 (1975), 269-274.

[8] M. Kac and P. van Moerbeke, A complete solution of the periodic Toda problem, Proc. Nat. Acad. Sci. USA 72 (1975), 2879-2880.

[9] E. Date and S. Tanaka, Analogue of inverse scattering theory for the discrete Hill equation and exact solutions for the periodic Toda lattice, Prog. Theor. Phys. 55 (1976), 457-465.

[10] A. V. Leznov and M. V. Saveliev, Representation of zero curvature for the system of nonlinear partial differential equations $x_{\alpha, z \bar{z}}=\exp (k x)_{\alpha}$ and its integrability, Lett. Math. Phys. 3 (1979), 489-494. 
[11] A. V. Mikhailov, Integrability of a two-dimensional generalization of the Toda chain, JETP Lett. 30 (1979), no. 7, 414-418.

[12] A. V. Mikhailov, The reduction problem and the inverse scattering method, Physica D3 (1981), 73-117.

[13] R. Hirota, Discrete analogue of a generalized Toda equation, J. Phys. Soc. Japan 50 (1981), 3785-3791.

[14] T. Miwa, On Hirota's difference equations, Proc. Japan Acad. 58, Ser. A (1982), 9-12.

[15] K. Ueno and K. Takasaki, Toda lattice hierarchy, K. Okamoto (ed.), Group Representations and Systems of Differential Equations, Advanced Studies in Pure Math. vol. 4, Kinokuniya, Tokyo, 1984, pp. 1-95.

[16] M. Sato and Y. Sato, Soliton equations as dynamical systems on infinitedimensional Grassmann manifold, H. Fujita, P.D. Lax and G. Strang (eds.), Nonlinear PDE in Applied Science, North-Holland, 1983, pp. 259-271.

[17] G. B. Segal and G. Wilson, Loop groups and equations of KdV type, Publ. Math. IHES 61 (1985), 5-65.

[18] M. Jimbo and T. Miwa, Solitons and infinite dimensional Lie algebras, Publ. RIMS Kyoto Univ. 19 (1983), 943-1001.

[19] M. Adler, On a trace functional for formal pseudo-differential operators and the symplectic structure of the Korteweg-deVries type equations, Invent. Math. 50 (1979), 219-248.

[20] E. Date, M. Jimbo, M. Kashiwara and T. Miwa, Operator approach to the Kadomtsev-Petviashvili equation - Transformation groups for soliton equations III —, J. Phys. Soc. Japan 50 (1981), 3806-3812.

[21] T. Takebe, Representation theoretical meaning of the initial value problem for the Toda lattice hierarchy I, Lett. Math. Phys. 21 (1991), 77-84.

[22] T. Takebe, Representation theoretical meaning of the initial value problem for the Toda lattice hierarchy II, Publ. RIMS Kyoto Univ. 27 (1991), 491-503.

[23] A. Alexandrov and A. Zabrodin, Free fermions and tau-functions, J. Geom. Phys. 67 (2013) 37-80. 
[24] K. Takasaki, Initial value problem for the Toda lattice hierarchy, K. Okamoto (ed.), Group Representations and Systems of Differential Equations, Advanced Studies in Pure Math. vol. 4, Kinokuniya, Tokyo, 1984, pp. 139-163.

[25] A. Gerasimov, A. Marshakov, A. Mironov, A. Morozov and A. Orlov, Matrix models of 2D gravity and Toda theory, Nucl. Phys. B357 (1991), 565-618.

[26] E. J. Martinec, On the origin of integrability in matrix models, Commun. Math. Phys. 138 (1991), 437-450.

[27] L. Alvarez-Gaumé, C. Gomez and J. Lacki, Integrability in random matrix models, Phys. Lett. B253 (1991), 56-62.

[28] S. Kharchev, A. Marshakov, A. Mironov and A. Morozov, Generalized Kontsevich model versus Toda hierarchy and discrete matrix models, Nucl. Phys. B397 (1993), 339-378.

[29] R. Dijkgraaf, G. Moore and R. Plesser, The partition function of 2D string theory, Nucl. Phys. B394 (1993), 356-382.

[30] A. Hanany, Y. Oz and R. Plesser, Topological Landau-Ginzburg formulation and integrable structure of 2d string theory, Nucl. Phys. B425 (1994), 150-172.

[31] T. Eguchi and H. Kanno, Toda lattice hierarchy and the topological description of $c=1$ string theory, Phys. Lett. B331 (1994), 330-334.

[32] K. Takasaki, Dispersionless Toda hierarchy and two-dimensional string theory, Comm. Math. Phys. 170 (1995), 101-116.

[33] T. Nakatsu, K. Takasaki and S. Tsujimaru, Quantum and classical aspects of deformed $c=1$ strings, Nucl. Phys. B443 (1995), 155-197.

[34] K. Takasaki, Toda lattice hierarchy and generalized string equations, Comm. Math. Phys. 181 (1996), 131-156.

[35] M. Adler and P. van Moerbeke, Matrix integrals, Toda symmetries, Virasoro constraints and orthogonal polynomials, Duke Math. J. 80 (1995), 863911.

[36] S. Kharchev, A. Mironov and A. Zhedanov, Faces of relativistic Toda chain, Int. J. Mod. Phys. A12 (1997), 2675-2724, 
[37] M. Adler and P. van Moerbeke, Integrals over classical groups, random permutations, Toda and Toeplitz lattices, Comm. Pure Appl. Math. 54 (2001), 153-205,

[38] M. J. Ablowitz and J. F. Ladik, Nonlinear differential-difference equations, J. Math. Phys. 16 (1975), 598-603.

[39] S. N. M. Ruijsenaars, Relativistic Toda systems, Commun. Math. Phys. 133 (1990), 217-247.

[40] K. Takasaki and T. Takebe, Quasi-classical limit of Toda hierarchy and W-infinity symmetries, Lett. Math. Phys. 28 (1993), 165-176.

[41] K. Takasaki and T. Takebe, Integrable hierarchies and dispersionless limit, Rev. Math. Phys. 7 (1995), 743-808.

[42] P.B. Wiegmann and A. Zabrodin, Conformal maps and integrable hierarchies, Comm. Math. Phys. 213 (2000), 523-538.

[43] M. Mineev-Weinstein, P.B. Wiegmann and A. Zabrodin, Integrable structure of interface dynamics, Phys. Rev. Lett. 84 (2000), 5106-5109.

[44] R. Pandharipande, The Toda equations and the Gromov-Witten theory of the Riemann sphere, Lett. Math. Phys. 53 (2000), 59-74.

[45] A. Okounkov, Toda equations for Hurwitz numbers, Math. Res. Lett. 7 (2000), 447-453.

[46] E. Getzler, The Toda conjecture, K. Fukaya et al. (eds.), Symplectic Geometry and Mirror Symmetry, World Scientific, 2001, pp. 51-79.

[47] A. Okounkov and R. Pandharipande, Gromov-Witten theory, Hurwitz theory, and completed cycles, Annals of Math. 163 (2006), 517-560.

[48] A. Okounkov and R. Pandharipande, The equivariant Gromov-Witten theory of $\mathbb{C P}^{1}$, Annals of Math. 163 (2006), 561-605.

[49] B. Dubrovin and Y. Zhang, Virasoro Symmetries of the Extended Toda Hierarchy, Comm. Math. Phys. 250 (2004), 161-193.

[50] W.-P. Li, Z. Qin and W. Wang, Hilbert schemes, integrable hierarchies, and Gromov-Witten theory, Intern. Math. Res. Notices 40 (2004), 20852104. 
[51] Z. Qin and W. Wang, Hilbert schemes of points on the minimal resolution and soliton equations, J. Lepowsky et al (eds.), Lie algebras, vertex operator algebras and their applications, Contemp. Math. vol. 442, Amer. Math. Soc., 2007, pp. 435-462.

[52] T. E. Milanov, The equivariant Gromov-Witten theory of $\mathbb{C P}^{1}$ and integrable hierarchies, Intern. Math. Res. Notices 2008 (2008), rnn073.

[53] A. Losev, A. Marshakov and N. Nekrasov, Small instantons, little strings and free fermions, M. Shifman, A. Vainstein and J. Wheater (eds.), From fields to strings: Circumnavigating theoretical physics, World Scientific, 2005, pp. 581-621.

[54] N. Nekrasov, Seiberg-Witten prepotential from instanton counting, Adv. Theor. Math. Phys. 7 (2004), 831-864.

[55] N. Nekrasov and A. Okounkov, Seiberg-Witten theory and random partitions, P. Etingof, V. Retakh and I. M. Singer (eds.), The unity of mathematics, Progr. Math. 244, Birkhäuser, 2006, pp. 525-296.

[56] A. Marshakov and N. Nekrasov, Extended Seiberg-Witten theory and integrable hierarchy, JHEP 0701 (2007), 104.

[57] A. Yu. Orlov and D. M. Scherbin, Fermionic representation for basic hypergeometric functions related to Schur polynomials, arXiv: nlin.SI/0001001.

[58] A. Yu. Orlov and D. M. Scherbin, Hypergeometric solutions of soliton equations, Theor. Math. Phys. 128 (2001), 906-926.

[59] A. Yu. Orlov and D. M. Scherbin, Multivariate hypergeometric functions as tau functions of Toda lattice and Kadomtsev-Petviashvili equation, Physica D: Nonlinear Phenomena 152-153 (2001), 51-65.

[60] T. Nakatsu and K. Takasaki, Melting crystal, quantum torus and Toda hierarchy, Comm. Math. Phys. 285 (2009), 445-468.

[61] T. Nakatsu and K. Takasaki, Integrable structure of melting crystal model with external potentials, M.-H. Saito et al (eds.), New developments in algebraic geometry, integrable systems and mirror symmetry, Advanced Studies in Pure Math. vol. 59, Math. Soc. Japan, 2010, pp. 201-223. 
[62] K. Takasaki and T. Nakatsu, Thermodynamic limit of random partitions and dispersionless Toda hierarchy, J. Phys. A: Math. Theor. 45 (2012), 025403 .

[63] K. Takasaki, Modified melting crystal model and Ablowitz-Ladik hierarchy, J. Phys. A: Math. Theor. 46 (2013), 245202.

[64] K. Takasaki, Orbifold melting crystal models and reductions of Toda hierarchy, J. Phys. A: Math. Theor. 48 (2015), 215201.

[65] A. Okounkov, N. Reshetikhin and C. Vafa, Quantum Calabi-Yau and classical crystals, P. Etingof, V. Retakh and I.M. Singer (eds.), The unity of mathematics, Progr. Math. vol. 244, Birkhäuser, 2006, pp. 597-618.

[66] T. Maeda, T. Nakatsu, K. Takasaki and T. Tamakoshi, Five-dimensional supersymmetric Yang-Mills theories and random plane partitions, JHEP 0503 (2005), 056.

[67] J. Bryan and R. Pandharipande, The local Gromov-Witten theory of curves, J. Amer. Math. Soc. 21 (2008), 101-136,

[68] N. Caporaso, L. Griguolo, M. Mariño, S. Pasquetti and D. Seminara, Phase transitions, double-scaling limit, and topological strings, Phys. Rev. D75 (2007), 046004,

[69] A. Brini, The local Gromov-Witten theory of $\mathbb{C P}^{1}$ and integrable hierarchies, Commun. Math. Phys. 313 (2012), 571-605,

[70] A. Brini, G. Carlet and P. Rossi, Integrable hierarchies and the mirror model of local $\mathbb{C P}^{1}$, Physica D241 (2012), 2156-2167.

[71] I. G. Macdonald, Symmetric functions and Hall polynomials, Oxford University Press, 1995.

[72] A. Yu. Orlov and E. I. Schulman, Additional symmetries for integrable equations and conformal algebra representation, Lett. Math. Phys. 12 (1986), 171-179.

[73] K. Takasaki, Generalized string equations for double Hurwitz numbers, J. Geom. Phys. 62 (2012), 1135-1156.

[74] K. Takasaki and T. Takebe, An $\hbar$-expansion of the Toda hierarchy: a recursive construction of solutions, Analysis and Mathematical Physics 2 (2012), 171-214. 
[75] M. Bruschi and O. Ragnisco, Lax representation and complete integrability for the periodic relativistic Toda lattice, Phys. Lett. A134 (1989), 365-370.

[76] V. Kac, Infinite Dimensional Lie algebras, Cambridge University Press, 1990.

[77] T. Miwa, M. Jimbo and E. Date, Solitons: Differential equations, symmetries, and infinite-dimensional algebras, Cambridge University Press, 2000 .

[78] S. Kharchev, A. Marshakov, A. Mironov and A. Morozov, Generalized Kazakov-Migdal-Kontsevich Model: group theory aspects, Int. J. Mod. Phys. A10 (1995), 2015.

[79] A. Orlov, Soliton theory, symmetric functions and matrix integrals, Acta Applicandae Mathematica 86 (2005), 131-158.

[80] A. Orlov, New solvable matrix integrals, Int. J. Mod. Phys. A19 (2004), 276-293.

[81] A. Orlov and T. Shiota, Schur function expansion for normal matrix model and associated discrete matrix models, Phys. Lett. A343 (2005), $384-396$.

[82] A. Alexandrov, Matrix models for random partitions, Nucl. Phys. B851 (2011) 620-650.

[83] A. Alexandrov, A. Mironov, A. Morozov and S. Natanzon, Integrability of Hurwitz partition functions. I. Summary, J. Phys. A: Math. Theor. 45 (2012), 045209.

[84] J. Harnad and A. Yu. Orlov, Hypergeometric $\tau$-functions, Hurwitz numbers and enumeration of paths, Comm. Math. Phys. 338 (2015), 267284.

[85] M. Guay-Paquet and J. Harnad, 2D Toda $\tau$-functions as combinatorial generating functions, Lett. Math. Phys. 105 (2015), 827-852.

[86] M. Guay-Paquet and J. Harnad, Generating functions for weighted Hurwitz numbers, J. Math. Phys. 58 (2017), 083503.

[87] J. Harnad, Multispecies quantum Hurwitz numbers, SIGMA 11 (2015), 097. 
[88] J. Harnad, Quantum Hurwitz numbers and Macdonald polynomials, J. Math. Phys. 57 (2016), 113505.

[89] B. Young (with an appendix by J. Bryan), Generating functions for coloured 3D Young diagrams and the Donaldson-Thomas invariants of orbifolds, Duke Math. J. 152 (2010), 115-153.

[90] L. Faddeev and A. Yu. Volkov, Abelian current algebra and the Virasoro algebra on the lattice, Phys. Lett. B315 (1993), 311-318.

[91] L. D. Faddeev and R. M. Kashaev, Quantum dilogarithm, Mod. Phys. Lett. A9 (1994), 427-434.

[92] A. Orlov, Hypergeometric tau functions $\tau\left(t, T, t^{*}\right)$ as $\infty$-soliton tau function in $T$ variables, arXiv:nlin.SI/0305001.

[93] E. Bettelheim, A. Abanov and P. Wiegmann, Nonlinear dynamics of quantum systems and soliton theory, J. Phys. A: Math. Theor. 40 (2007), F193-F208.

[94] K. Takasaki and T. Nakatsu, Open string amplitudes of closed topological vertex, J. Phys. A: Math. Theor. 49 (2016), 025201. 